\title{
A Monocular Vision-Based Framework for Power Cable Cross-Section Measurement
}

\author{
Xiaoming Zhang ${ }^{1,2} \mathbb{D}$ and Hui Yin ${ }^{1,2, *}$ \\ 1 School of Geodesy and Geomatics, Wuhan University, No.129, Luoyu Road, Wuhan 430079, China \\ 2 Collaborative Innovation Center for Geospatial Technology, No.129, Luoyu Road, Wuhan 430079, China \\ * Correspondence: hyin@sgg.whu.edu.cn
}

Received: 6 June 2019; Accepted: 2 August 2019; Published: 6 August 2019

\begin{abstract}
The measurements of the diameter of different layers, the thickness of different layers and the eccentricity of insulation layer in the cross-section of power cables are important items of power cable test, which currently depend on labor-intensive manual operations. To improve efficiency, automatic measurement methods are in urgent need. In this paper, a monocular vision-based framework for automatic measurement of the diameter, thickness, and eccentricity of interest in the cross-section of power cables is proposed. The proposed framework mainly consists of three steps. In the first step, the images of cable cross-section are captured and undistorted with the camera calibration parameters. In the second step, the contours of each layer are detected in the cable cross-section image. In order to detect the complete and accurate contours of each layer, the structural edges in the cross-section image are firstly detected and divided into individual layers, then unconnected edges are connected by arc-based method, and finally contours are refined by the proposed break detection and grouping (BDG) and linear trend-based correction (LTBC) algorithm. In the third step, the monocular vision-based cross-section dimension measurement is accomplished by placing a chessboard coplanar with the power cable cross-section plane. The homography matrix mapping pixel coordinates to chessboard world coordinates is estimated, and the diameter, thickness and eccentricity of specific layers are calculated by homography matrix-based measurement method. Simulated data and actual cable data are both used to validate the proposed method. The experimental results show that diameter, minimum thickness, mean thickness and insulation eccentricity of simulated image without additive noise are measured with root mean squared error (RMSE) of $0.424,0.103$ and $0.063 \mathrm{~mm}$, and 0.002 , respectively, those of simulated image with additive Gaussian noise and salt and pepper noise are measured with RMSE of $0.502,0.243$ and $0.058 \mathrm{~mm}$ and 0.001 . Diameter, minimum thickness and mean thickness of actual cable images are measured with average RMSE of $0.768,0.308$ and $0.327 \mathrm{~mm}$. The measurement error of insulation eccentricity of actual cable image is comparatively large, and the measurement accuracy should be improved.
\end{abstract}

Keywords: power cable test; cross-section dimension measurement; contour detection; defect detection; monocular vision-based measurement

\section{Introduction}

High voltage power cables are used for power transmission and distribution in power systems, and cable performance test is essential to reduce operational accidents and improve power supply reliability. Measurements of electrical properties, thermal properties, mechanical properties and different geometric dimensions in the cross-section are included in the power cable test [1-4]. The defeat of power cables in geometric dimensions will lead to severe electrical and mechanical performance loss, and the accurate measurement of geometric dimensions are of great importance to power cable defect detection. 
The geometric dimension measurements of power cables mainly involve the diameter and thickness of different layers and the eccentricity of insulation layer in the cross-section. The traditional manual measurement methods of cable dimensions are kinds of destructive test, in which each layer of interest is measured according to specifications [1-4] after specimen preparations such as cutting, stripping layer by layer, cleanout, and slicing. Take the power cable with Cross-Linked Polyethylene (XLPE) insulation for rated voltage of $110 \mathrm{kV}$ as an example, the specimen is first stripped layer by layer, diameters up to $25 \mathrm{~mm}$ are directly measured with a micrometer or a vernier caliper reading to $0.01 \mathrm{~mm}$, and diameters more than $25 \mathrm{~mm}$ are calculated from perimeter measured with a steel tape reading to $0.1 \mathrm{~mm}$. The thickness of nonmetal and metal sheath is measured with a micrometer reading to $0.01 \mathrm{~mm}$. The three insulation related layers are cleaned and sliced first and the thickness is measured with a digital projector or a microscope reading to $0.01 \mathrm{~mm}$.

Diverse manual measuring operations and specimen preparations are involved in traditional measurement methods, which are labor-intensive and inefficient. Besides, the dimension measured by different operators may be different due to inevitable personal errors. Automatic methods for dimension measurements in power cable cross-section are starved for. Current automatic measurement methods mainly include radiation method [5-9], electromagnetic induction method [10,11], and photoelectricity method (vision-based method) [12-15]. In radiation methods, diameter and thickness of insulation layer and nonmetal sheath layer can be measured online directly with X-ray penetrating the multilayer specimen. There are mature online $\mathrm{X}$-ray measuring systems $[5,6]$, but they are only designed for one insulation layer or three insulation-related layers, and the $X$-ray apparatus are of high costs. When used for multilayer modeling, the contours of all layers are theoretically treated as eccentric circles [9], so the measuring accuracy is very limited. For electromagnetic induction methods, Wen and Zhao [11] measured the thickness and eccentricity of insulation layer with an eddy current sensor, and Liang et al. [10] measured the thickness of aluminum sheath with two eddy current sensors of high and low frequency excitation. Although multilayer specimen is allowed in electromagnetic induction method, this metal-sensitive measurement method is designed only for a single layer, and the outmost diameter is generally measured by the photoelectric method. In photoelectric methods, the image of insulation layer $[12,13]$ or nonmetal sheath layer $[14,15]$ slice is first acquired by digital microscope or camera, then contours are detected in the image, and the diameter and thickness are finally calculated according to an amplification factor calibrated by a ruler. The existing photoelectric methods are only suitable for cleaned specimen slices, in which cleaning and slicing of specimen are tedious.

Vision-based methods are more and more used for automatic and efficient defect detection and dimension measurement in engineering. Cheng et al. [16] proposed a detection and localization method for self-shattering defects detection in twin glass insulators based on spatial features. Siddiqui et al. [17] utilized a convolutional neural networks based method to automatic powerline equipment detection, and a novel rotation normalization and ellipse detection algorithm was used for insulator detection. Hao et al. [18] conducted image segmentation of ice-covered insulators using proposed GrabCut algorithm, and then calculated the shed overhang and shed spacing with contour convexity defect recognition for icing condition assessment. Fard et al. [19] monitored the electrical tree (ET) in XLPE cable insulation with a camera attached to the microscope and studied the effect of different voltage types on ET and partial discharge. Kang et al. [20] extracted the boundary of a burning wood pellet using scanning lines and then calculated its volume based on radius and height, which is important to understand the wood pellet burning mechanism. Lee et al. [21] proposed a vision-based method with an adaptive region-of-interest algorithm to locate the marker attached to bridge, and the structural displacements of bridge were measured based on planar homography. Zhang et al. [22] measured the steel bar diameter, spacing, and quantity by sub-pixel boundary location in smartphone image and fast image stitch.

In the geometric dimension measurements of power cable, current vision-based automatic methods are mainly used for the sliced insulation related layers or nonmetal sheath layer, and measuring 
methods for other layers have been rarely reported. Since the vision-based method can obtain a complete cross-section image of power cable along the axial direction, it has a large potential to simultaneously measure the diameter and thickness of different layers and the eccentricity of insulation layer. This paper tries to develop a monocular vision-based method to measure the diameter, thickness, and eccentricity of interest in the cable cross-section. There are single-core and multi-core power cables, in which core means the innermost solid or stranded conductor. Since single-core structure is the most common type and the basis of the multi-core structure, this paper solely deals with the automatic cross-section measurement of single-core power cable.

This paper proposes a monocular vision-based framework to automatically measure the cross-section dimensions of power cable. In our framework, the camera is first calibrated and the distortion parameters are used to undistort the images of power cable cross-section. The next key step of this vision-based method for power cable cross-section measurement is contour detection of different layers. Circle detection methods such as improved Hough Transform methods [23-25], geometric feature methods [26-28], template matching method [29-31], and optimization method [32,33] are usually used to detect such circular contours. Due to the existence of global deformations and local defects in the multilayer contours of cross-section, current circle detection methods cannot meet the demands of contour detection in power cable cross-section measurement. In order to accurately and completely detect the contour of each layer, contours of metal layers are firstly extracted in this paper and used as a reference for following operations, then the structural edges in the cross-section are detected and divided into individual layers. The arc-based method is used to connect the discontinuous edges in each layer. Finally, the contours are refined by proposed break detection and grouping (BDG) and linear trend-based correction (LTBC) algorithm. In the last step, geometric dimension measurements based on monocular vision are accomplished by placing a chessboard coplanar with the power cable cross-section plane. The homography matrix mapping pixel coordinates to chessboard world coordinates is firstly estimated. With the detected contours of each layer, the diameter and thickness of different layers and the eccentricity of insulation layer are then calculated by mapping the contour pixels to chessboard world coordinates based on the estimated homography matrix, which is more logically rigorous than conversion with an amplification factor.

This paper is organized as follows: Section 2 introduces the framework we developed, including image undistortion, multilayer contour detection in cable cross-section, and cross-section dimension measurement. Section 3 demonstrates and analyzes the cross-section measurement results of both simulated data and actual cable specimen data, and discusses the influence of printed chessboard on measurement accuracy. Section 4 presents conclusions and future works.

\section{Methodology}

Figure 1 shows a typical single-core power cable cross-section, and each contour is denoted by $C_{k}(k=12,23,34,45,56,67,78,80)$, where the subscript $k$ indicates its adjacent structural layers. For instance, $C_{12}$ is interface between layers 1 and 2, and the special $C_{80}$ is outer contour of layer 8 . The innermost layer 1 denotes stranded copper conductor with rich chrominance information due to its material. Layer 2 consists of wrapped semi-conductive tape with partial overlapping, which is used to tighten the conductor and almost invisible to naked eyes in cross-section. In order to express the existence of layer 2, contour $C_{23}$ is exaggeratedly drawn in Figure 1. Layers 3-5 are conductor screen, XLPE insulation, and insulation screen, which are produced by the three-layer co-extrusion method with high roundness and tightness. Layer 6 is wrapped semi-conductive buffer water block, layer 7 is flexible corrugated aluminum sheath, and layer 8 is nonmetal outer sheath. The material and manufacturing techniques of each layer determine that inner layers 1-5 are less deformed and good concentric shapes are maintained. The outer layers 6-8 may have larger deformation under extrusion. 


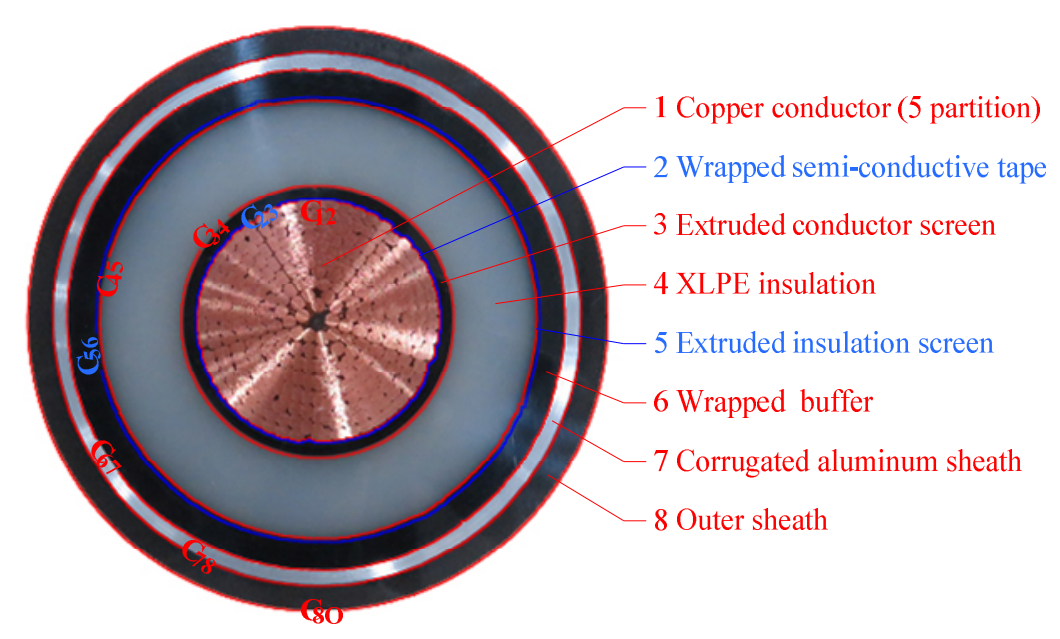

Figure 1. Cross-section of the structure of typical single-core cable.

According to standards [1-4], diameter $D_{i}$ of layer $(i=1,5,6,7,8)$, minimum thickness $\left(t_{i}\right)_{\text {min }}$ and mean thickness $\bar{t}_{i}$ of layer $i(i=3,4,5,7,8)$, and eccentricity $e_{4}$ of insulation layer (layer 4$)$ should be measured. So, contour extraction of all layers as much as possible is required in image processing for the cross-section measurement. Since the wrapped tape is almost invisible in cable cross-section, $C_{23}$ cannot be extracted in this vision-based method and the calculated thickness of conductor screen (layer 3 ) in actual power cable cross-section actually contains wrapped tape (layer 2) and conductor screen. The detection of $C_{56}$ between insulation screen (layer 5) and wrapped buffer (layer 6) is challenging as it is in the similar background and near $C_{45}$.

The flowchart of the proposed framework is shown in Figure 2. This vision-based framework for power cable cross-section measurement mainly contains three steps: image undistortion, multilayer contour detection in cable cross-section, and cross-section dimension measurement. In the first step, the camera is calibrated and captured images of power cable cross-section are undistorted with the camera calibration parameters. In the second step, structural edges in the cross-section are firstly detected and divided into individual layers, unconnected edges are then connected by arc-based method, and contours are further refined by BDG and LTBC algorithm. In the third step, the diameter and the thickness of different layers and the eccentricity of insulation layer are calculated by mapping the detected contour pixels to chessboard world coordinates based on homography matrix $H$.
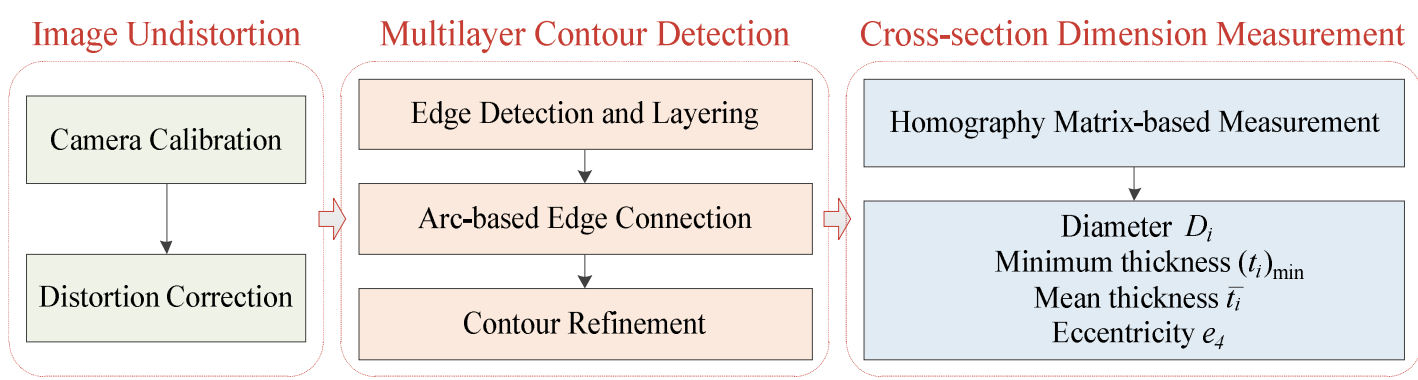

Figure 2. Flow diagram of the proposed vision-based framework for power cable cross-section measurement.

\subsection{Image Undistortion}

The camera calibration is conducted with a chessboard. As shown in Figure 3, the world coordinate system $O_{w}-X_{w} Y_{w} Z_{w}$ is a left-handed coordinate system with the origin $O_{w}$ at the upper left chessboard corner, $X_{w}$ axis to the right and $Y_{w}$ axis down. The pixel coordinate system is denoted by $o-u v$, and image coordinate system $o_{\mathcal{c}}-x y$ is established with the origin $o_{\mathcal{c}}$ at the principal point, $x$ axis parallel to 
the horizontal sampling direction of pixel ( $u$ axis), and $y$ axis parallel to the vertical sampling direction ( $v$ axis).

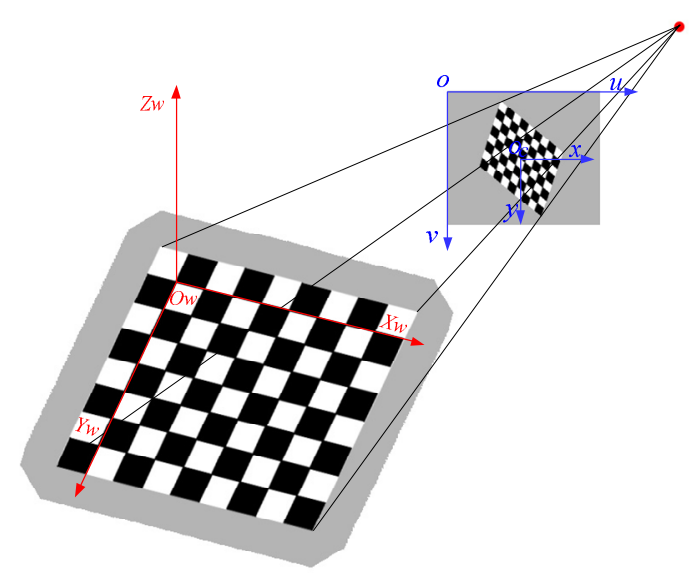

Figure 3. Coordinate systems.

When we use the chessboard for calibration, arbitrary point $P$ on the chessboard is in the defined $O_{w} X_{w} Y_{w}$ plane with $Z_{w}=0$. Mapping relationship from spatial point $P$ to image point $p$ is shown as Equation (1):

$$
s\left[\begin{array}{c}
u \\
v \\
1
\end{array}\right]=M\left[\begin{array}{ll}
R & T
\end{array}\right]\left[\begin{array}{c}
X_{w} \\
Y_{w} \\
0 \\
1
\end{array}\right]=M\left[\begin{array}{lll}
r_{1} & r_{2} & T
\end{array}\right]\left[\begin{array}{c}
X_{w} \\
Y_{w} \\
1
\end{array}\right],
$$

where $s$ is an arbitrary scale factor, $M=\left[\begin{array}{ccc}f_{u} & 0 & u_{0} \\ 0 & f_{v} & v_{0} \\ 0 & 0 & 1\end{array}\right]$ is the intrinsic matrix for camera, $R$ is a 3-by-3 rotation matrix which can be broken up into three 3-by-1 columns $\left(R=\left[\begin{array}{lll}r_{1} & r_{2} & r_{3}\end{array}\right]\right)$, and $T$ is a 3-by-1 translation matrix.

Equations (2) and (3) shows the model [34] considering radial distortion and tangential distortion:

$$
\begin{gathered}
u^{\prime}=u+\bar{u}\left(k_{1} r^{2}+k_{2} r^{4}+k_{3} r^{6}\right)+\left[2 p_{1} \overline{u v}+p_{2}\left(r^{2}+2 \bar{u}^{2}\right)\right] \\
v^{\prime}=v+\bar{v}\left(k_{1} r^{2}+k_{2} r^{4}+k_{3} r^{6}\right)+\left[p_{1}\left(r^{2}+2 \bar{v}^{2}\right)+2 p_{2} \overline{u v}\right] \\
\bar{u}=u-u_{0} \\
\bar{v}=v-v_{0} \\
r^{2}=\bar{u}^{2}+\bar{v}^{2}
\end{gathered}
$$

where $\left(u^{\prime}, v^{\prime}\right)$ are distorted pixel coordinates, $(u, v)$ are undistorted pixel coordinates, $k_{i}(i=1,2,3)$ denotes radial distortion parameter, and $p_{i}(i=1,2)$ denotes tangential distortion parameter.

The distortion is firstly assumed to 0 , and the initial solution to intrinsic and extrinsic parameters are obtained by Zhang's method [35]. Then the radial and tangential distortions are considered on the basis of the pinhole model. The final parameters are acquired by Levenberg-Marquardt iterative algorithm based on maximum likelihood criterion [36,37]. Then undistorted images of power cable cross-section can be obtained by distortion correction based on camera calibration parameters.

\subsection{Multilayer Contour Detection in Cable Cross-Section}

The aim of multilayer contour detection is to get continuous and accurate contour of each layer in the cross-section. Structural edges in the cross-section are first detected and divided into individual 
layers. Edges in each layer are denoted by $\operatorname{Edg} e_{k}(k \in\{12,23,34,45,56,67,78,80\})$, in which $k$ indicates its adjacent structural layers. Since edges in a layer may be discontinuous, the unconnected parts in $E d g e_{k}$ are then connected by arc-based method to get the initial closed contour $C_{k}$. To solve the problem of possible mutations in initial contour caused by interference edges or inaccurate edge connection, each initial contour $C_{k}$ is refined to final contour by the proposed BDG and LTBC methods.

\subsubsection{Edge Detection and Layering}

The contours of metal layers are much easier to extract, so the coarse contours of metal layers, including copper conductor (layer 1) and aluminum sheath layer (layer 7), are firstly extracted based on thresholding. Similar to contour $C_{k}$, notation $r C_{k}(k=12,67,78)$ is used to denote the coarse contours of metal layers, and the pixel length of $r C_{k}$ is denoted by $r L_{k}$. The copper conductor is segmented based on chrominance information in $U^{\prime}$ component, and the coarse contour $r C_{12}$ is extracted with the length of $r L_{12}$. In YUV color space, $U$ is blue component offset from brightness [38], so $U^{\prime}=-U$ can be seen as yellow component offset from brightness. According to the definitions, Equation (7) can be derived, where constant $U_{\max }$ determines $U^{\prime} \in\left[-U_{\max }, U_{\max }\right]$. The coefficients of $R, G$, and $B$ sum to 0 , which ensures $U^{\prime} \approx 0$ when $R \approx G \approx B$ and can be used to weaken the background in $U^{\prime}$ component:

$$
U^{\prime}=U_{\max }(\alpha R+(1-\alpha) G-B)
$$

After the measurement image is converted to grayscale image $I G$, the aluminum sheath is segmented based on brightness information in $I G$, and coarse contours $r C_{78}$ and $r C_{67}$ are then extracted. The coarse contours conform to the actual structural contours but not accurate enough, and they are used as a reference for following line edge detection.

Candidate edges are acquired through Canny edge detection [39] in grayscale image IG, including actual structural edge, details inside each structural layer, stain, and cutting texture, which should be further filtered based on the conditions of length and linearity features.

As shown in Figure 4, candidate edges can be firstly divided into three categories based on length. (1) Taking the length of innermost coarse contour $r C_{12}$ as reference, the long edges with length no less than $t_{l} \cdot r L_{12}$ are considered as reliable structural edge (Figure $\left.4 \mathrm{a}\right) . t_{l}$ is a proportion of length threshold to the coarse contour length $r L_{12}$ of copper conductor. (2) The very short edges with length no more than $t_{s}$ pixels are regarded as discrete interference details (Figure $4 \mathrm{~b}$ ) and directly removed. (3) The left edges in Figure $4 \mathrm{c}$ contain both structural edges and interference details, pending to further linearity judgment. To describe the edge linearity, the eccentricity of ellipse that has the same second moment as $i$-th pending edge is calculated and denoted by $e_{i}^{\prime}$. The value of $e^{\prime}$ ranges from 0 to 1 : the closer $e^{\prime}$ is to 0 , the closer the shape is to a circle; the closer $e^{\prime}$ is to 1 , the closer the shape is to a line. Short structural edges with fairly good linearity (Figure 4 d) are then extracted from pending edges based on the condition of $e_{i}^{\prime} \geq e_{\min }^{\prime}$. The whole structural edges in cable cross-section are made up of short structural edges with fairly good linearity and the previous long structural edges.

The structural edges are then divided into individual layers with result of $E d g e_{k}$, in which $k \epsilon\{12,23,34,45,56,67,78,80\}$ indicates its adjacent structural layers. The outermost edge $E d g e_{80}$ is firstly obtained by hole filling of structural edges. With the previous extracted location of coarse contours $r C_{k}, \operatorname{Edge}_{k}(k=12,67,78)$ is then successively extracted from structural edges. The left structural edges involve layers of wrapped tape (layer 2), conductor screen (layer 3), XLPE insulation (layer 4), insulation screen (layer 5), and wrapped buffer (layer 6), which should be further divided into specific layers. Considering that contours of inner layers 1-5 are less deformed and maintain a good concentric shape, edge points in an individual layer are of nearly equal distances from the approximate center. Therefore, the edge points can be divided into specific layers in polar coordinate system by annulus division according to the polar radius. The annulus with structural edge $E d g e_{k}(k \epsilon\{23,34,45,56\})$ can be extracted based on two characteristics: (1) points in this annulus are far more than those in other annuli with a few useless details; (2) polar angles of points in this annulus are in a larger range 
than those in other annuli with local useless details. Points in each extracted $E d g e_{k}$ are fitted to an approximate circular contour $C_{c k}$ for further use. The center, radius, and maximum fitting error of fitted circle $C_{c k}$ are denoted by $O_{c k}, R_{c k}$, and $\sigma_{k}$ respectively.

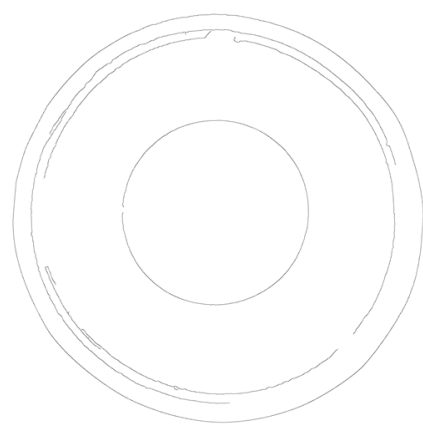

(a)

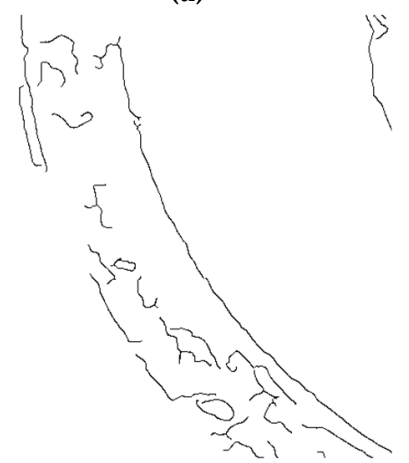

(c)

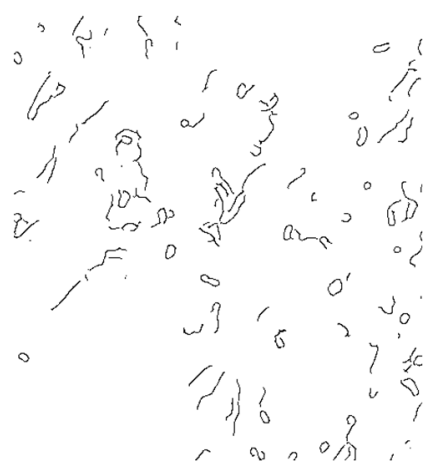

(b)
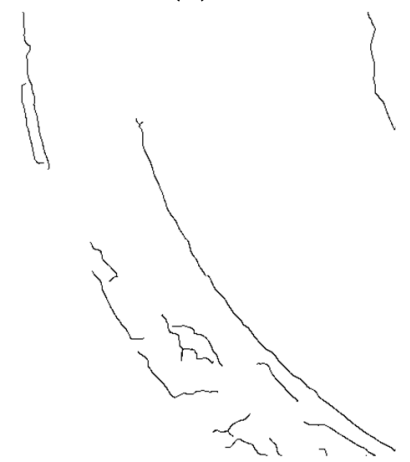

(d)

Figure 4. Edge filtering based on length and linearity. (a) Long structural edges; (b) Very short interference details; (c) Pending short edges; (d) Short structural edges with fairly good linearity.

\subsubsection{Arc-Based Edge Connection}

Affected by local interferences, the detected structural edges in a layer may be discontinuous, and unconnected edges in each $E d g e_{k}$ should be connected to get a closed contour. Structural edges in each $E d g e_{k}$ are connected by the proposed arc-based method of direct connection or prediction-and-search connection (PSC) with local constraints of fitted circular contour $C_{c k}$. All structural edges in each $E d g e_{k}$ are firstly clockwise organized as $\operatorname{Arc}_{p}(p=1,2,3 \ldots)$, and points in each arc are also clockwise arranged with start point of $P_{p \mathrm{~S}}$ and end point $P_{p \mathrm{E}}$. The arc-based edge connection starts with $P_{1 \mathrm{E}}$ to the next $P_{2 S}$ as follows.

As shown in Figure 5a, red dots are edge points to be connected, green line denotes fitted circular contour $C_{c k}$, and direct connection is first tried through creating a connecting line $P_{1 \mathrm{E}} P_{2 S}$. Then distance $\varepsilon_{i}$ between each point in $P_{1 \mathrm{E}} P_{2 S}$ and $C_{c k}$ is calculated, and root mean squared error (RMSE) is calculated by Equation (8) and used to measure the agreement degree of $P_{1 \mathrm{E}} P_{2 S}$ and $C_{c k}$ :

$$
s_{c}=\sqrt{\sum_{i=1}^{N} \varepsilon_{i}{ }^{2} / N} .
$$




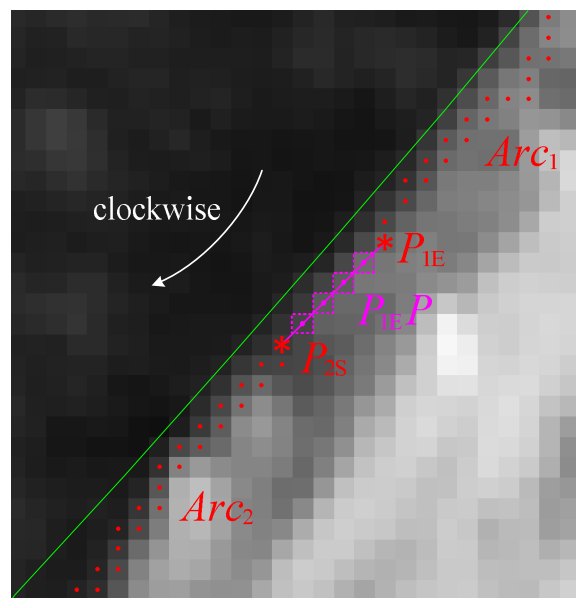

(a)

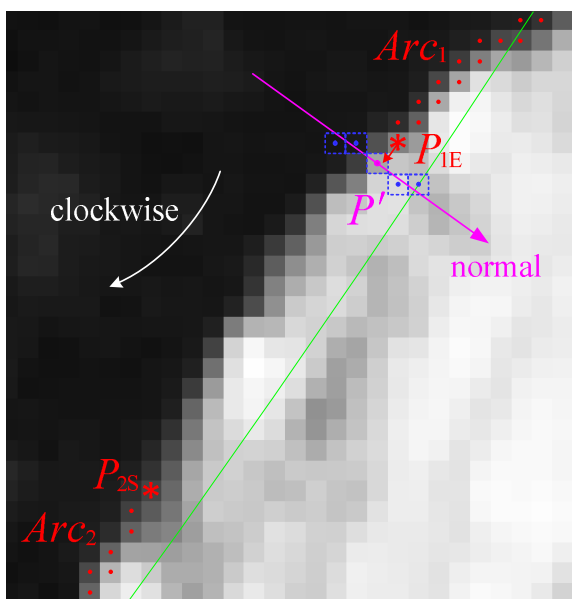

(b)

Figure 5. Arc-based edge connection, in which red dots are clockwise organized edge points to be connected, red asterisks denote arc endpoints, and green line denotes the fitted circular contour $C_{c k}$. (a) Direct connection with connecting line $P_{1 \mathrm{E}} P_{2 S}$; (b) PSC method, in which red arrow shows the tangential direction of $C_{c k}$ at $P_{1 \mathrm{E}}, P^{\prime}$ denotes the prediction point, magenta arrow shows the normal direction of $C_{c k}$ at $P^{\prime}$, and blue boxes indicate the search range for gradient calculation.

In Equation (8), $N$ is the number of points in line $P_{1 E} P_{2 S} . s_{\mathcal{C}} \leq \max \left(\varepsilon_{1 E}, \varepsilon_{2 S}\right)$ means the distance of $P_{1 \mathrm{E}} P_{2 S}$ from $C_{c k}$ does not exceed that of endpoints, $P_{1 \mathrm{E}} P_{2 \mathrm{~S}}$ agrees well with $C_{c k}$, and $\operatorname{Arc} c_{1}$ is updated with connected $A r c_{1}, P_{1 E} P_{2 S}$, and $A r c_{2}$. Otherwise $s_{c}>\max \left(\varepsilon_{1 E}, \varepsilon_{2 S}\right)$ indicates a large deviation of line $P_{1 \mathrm{E}} P_{2 S}$ from $C_{c k}$, and the proposed PSC method will be considered.

The idea of PSC is to find appending edge points in local search range with maximum gradient projection in the normal direction. As shown in Figure 5b, red arrow shows the tangential direction of $C_{c k}$ at $P_{1 E}$, and a prediction point $P^{\prime}$ is set to the next point in this tangential direction. Magenta arrow shows the normal (radial) direction of $C_{c k}$ at $P^{\prime}$, and the appending point is then searched in this normal direction around $P^{\prime}$. Gradient and its direction of image $I G$ in search range are calculated, and the point with maximum gradient projection in the normal direction is appended to initial $A r c_{1}$. Repeat the steps of appending point determination until $A r c_{1}$ is connected to $A r c_{2}$, and $A r c_{1}$ is updated with the connected $A r c_{1}$ and $A r c_{2}$.

After $A r c_{2}$ is brought into $A r c_{1}$, the next arc connection is continued with above-mentioned direct connection or PSC, until all arcs are contained in $A r c_{1}$ and $A r c_{1}$ is closed. The closed $A r c_{1}$ after edge connection is denoted by initial contour $C_{k}(k \epsilon\{12,23,34,45,56,67,78,80\})$.

\subsubsection{Contour Refinement}

The initial contour $C_{k}$ is not smooth enough and fluctuations exist in initial contour $C_{k}$. As shown in Figure 6, red dots are extracted initial contour, green line denotes the fitted circular contour $C_{c k}$, and fluctuations exist in the blue ellipse. The fluctuations in initial $C_{k}$ include structure-related gentle changes (Figure 6a) and mutations caused by interference edges and inaccurate edge connection (Figure 6b), in which mutations with steep changes should be distinguished and refined. Since mutations occur at locations with a large radial change in a very small tangential range, the mutations can be detected in the polar coordinate system with pole at the center $O_{c k}$ of fitted circle. 


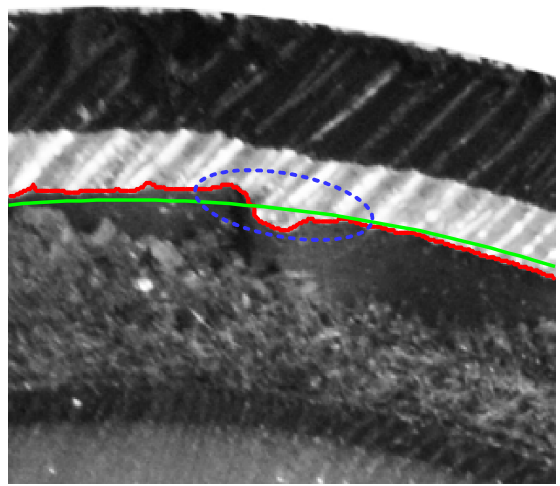

(a)

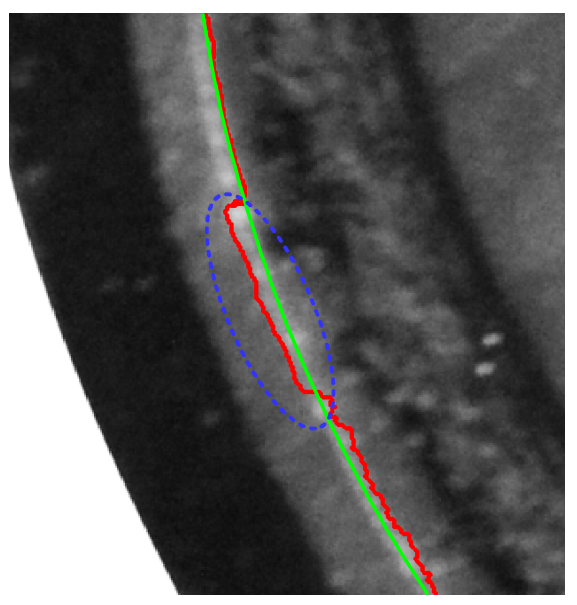

(b)

Figure 6. Fluctuations in initial contour $C_{k}$, in which red dots are contour points, green line denotes the fitted circular contour $C_{c k}$, and fluctuations exist in blue ellipse. (a) Structure-related gentle changes; (b) Mutations with steep changes.

The $i$-th point $P_{i}$ in contour $C_{k}$ is firstly converted to polar coordinates $\left(\rho_{i}, \theta_{i}\right)$, and the radial distance from this point to the fitted circle $C_{c k}$ is denoted as $\left(V_{\rho}\right)_{i}$ and calculated by Equation (9):

$$
\left(V_{\rho}\right)_{i}=\rho_{i}-R_{c k}, \quad i=1,2,3 \ldots,
$$

where $R_{c k}$ is radius of the fitted circle $C_{c k}$.

In Figure 7, the vertical axis denotes radial distance $V_{\rho}$ to the fitted circle, horizontal axis denotes polar angle $\theta$ in degrees, and the $V_{\rho}(\theta)$ sequence is drawn in green line. Similar to a pulse in signal processing, the mutation in $V_{\rho}(\theta)$ is defined as a $B_{1}-S_{t e p_{\mathrm{b}}}-B_{2}$ (B-S-B) structure in Figure 7, in which break $B_{1}$ indicates the beginning segment of steep change away from the overall trend, break $B_{2}$ indicates the ending segment of steep changes back to the overall trend, and $S t e p_{\mathrm{b}}$ denotes the sequence between $B_{1}$ and $B_{2}$. If Step $\mathrm{p}_{\mathrm{b}}$ contains several points, this B-S-B structure is an interval type mutation (I-Mutation) (magenta arrow in Figure 7a); otherwise, $S$ te $p_{\mathrm{b}}$ is the intersection point of $B_{1}$ and $B_{2}$, and this B-S-B structure is a point type mutation (P-Mutation) (orange arrow in Figure 7b). Step 0 denotes the point sequence between two adjacent mutations. Mutations in $V_{\rho}(\theta)$ are detected and corrected by BDG and LTBC algorithm. The BDG algorithm is designed for breaks $\left(B_{1}\right.$ and $\left.B_{2}\right)$ detection, breaks pairing and mutation grouping. To detect the breaks, $V_{\rho}(\theta)$ sequence is firstly smoothed and simplified by Douglas-Peuker (DP) decimation [40] to keep only major fluctuations, and the result is denoted by $D P(\theta)$ and drawn with red dashed line in Figure 7 . The absolute radial difference of two consecutive points in simplified $D P(\theta)$ is then calculated by Equation (10):

$$
\left|\mathrm{d} D P\left(\theta_{j}\right)\right|=\left|D P\left(\theta_{j}\right)-D P\left(\theta_{j-1}\right)\right|, \quad j \in i \text {. }
$$




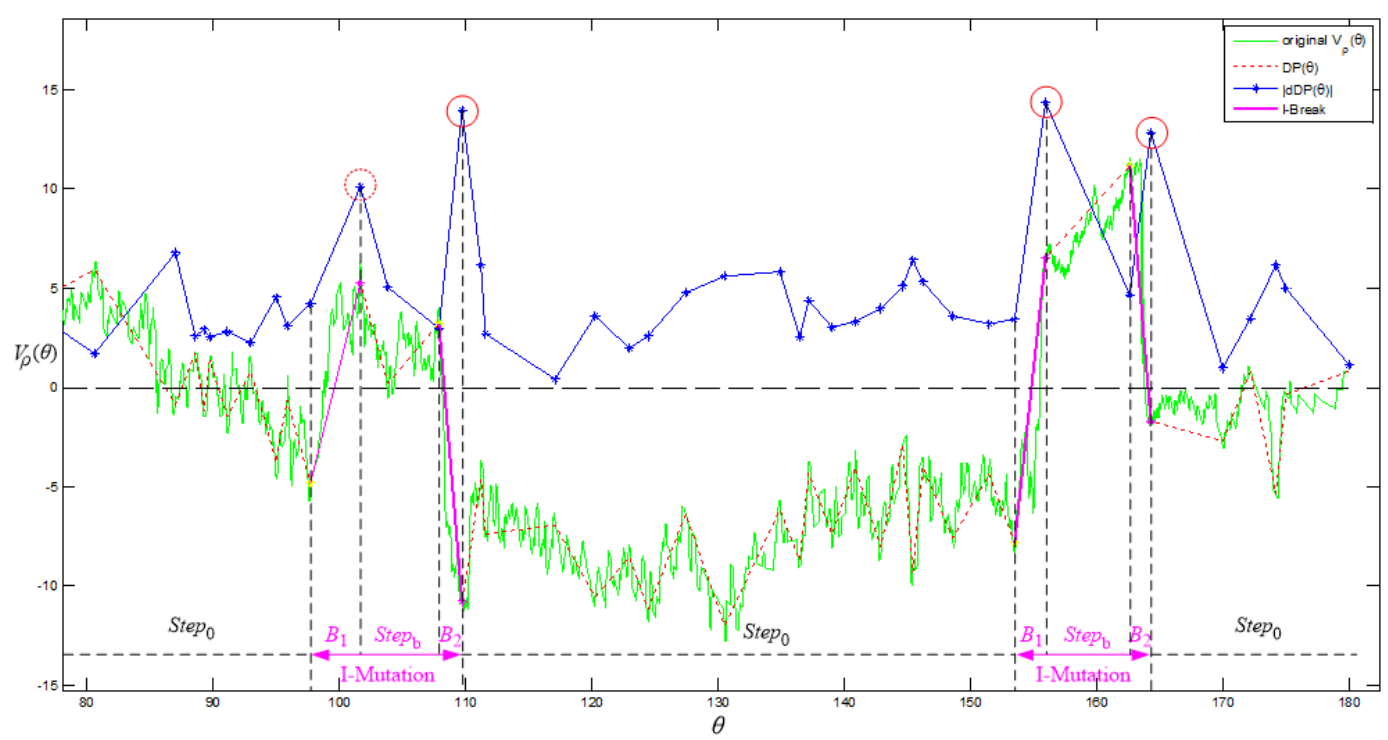

(a)

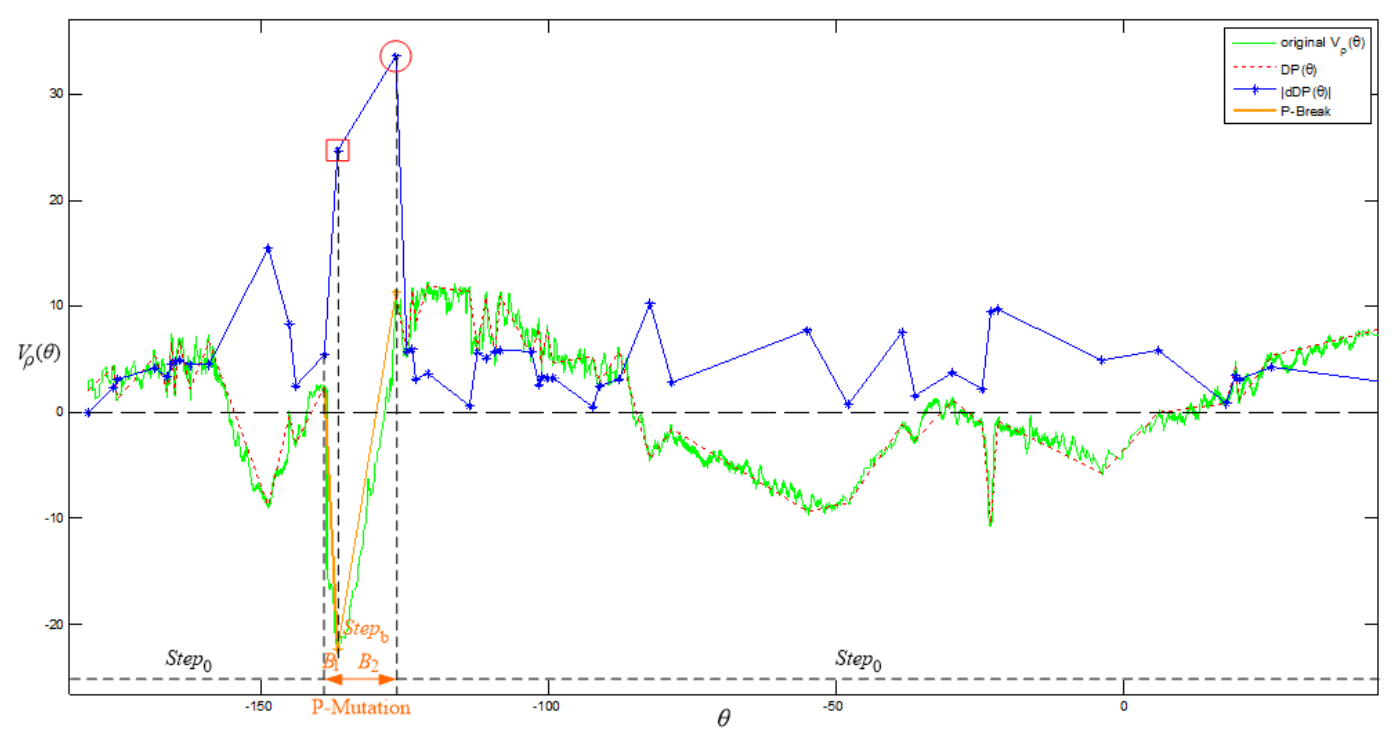

(b)

Figure 7. Breaks detection and mutation grouping, in which horizontal axis is polar angle $\theta$, vertical axis is radial distance to the fitted circle, green solid line denotes original $V_{\rho}(\theta)$ sequence, red dashed line denotes the simplified $D P(\theta)$ sequence, blue line is the absolute difference of $D P(\theta)$, and the segments divided by break groups are labeled. (a) I-Break in magenta lines with detected major peaks in circles; (b) P-Break in orange lines with a detected major peak in circle.

The $|\mathrm{d} D P(\theta)|$ sequence is used to quantize the radial change of points in $D P(\theta)$ and drawn with blue line in Figure 7. As shown in Figure 7, a break in $V_{\rho}(\theta)$ is a sequence segment with steep changes, and it appears in the form of a peak point (local maxima) in $|\mathrm{d} D P(\theta)|$ sequence. So, all points in $|\mathrm{d} D P(\theta)|$ sequence that are larger than their two neighboring points are obtained as peaks and breaks are further detected from these peaks. As shown in Figure 8, breaks can be detected based on $|\mathrm{d} D P(\theta)|$ and the angle $\alpha$ between two consecutive points and radial direction. In Figure 8 , red dots are continuous contour points in the original $V_{\rho}(\theta)$ sequence, point $P_{j-1}$ and $P_{j}$ denote two consecutive points in the simplified $D P(\theta)$ sequence, $\mathrm{d} D P\left(\theta_{j}\right)$ and $\mathrm{d} \theta_{j}$ are the radial and angular difference between point $P_{j}$ and $P_{j-1}$, and $\alpha$ is the angle between line $P_{j-1} P_{j}$ and radial direction at 
point $P_{j}$. The $V_{\rho}(\theta)$ sequence between point $P_{j-1}$ and $P_{j}$ is determined as a break if $\left|\mathrm{d} D P\left(\theta_{j}\right)\right| \geq t_{\rho}$ and $\alpha<t_{\alpha}$. Peaks in $|\mathrm{d} D P(\theta)|$ meeting the above two conditions are extracted as major peaks (red circle in Figure 7), and the corresponding breaks in $V_{\rho}(\theta)$ are obtained. After breaks are detected, each detected break should be paired with another break to make up a group of B-S-B structure. Since the breaks $B_{1}$ and $B_{2}$ in a pair are steep changes away from and back to the overall trend respectively, the differences $\mathrm{d} D P(\theta)$ corresponding $B_{1}$ and $B_{2}$ must be in opposite signs. Paired P-breaks are first detected from the points adjacent to major peaks in $|\mathrm{d} D P(\theta)|$ sequence. For a major peak at point $P_{j}$, check whether point $P_{j-1}$ or $P_{j+1}$ meets the condition of $|\mathrm{d} D P(\theta)| \geq t_{p} \cdot\left|\mathrm{d} D P\left(\theta_{j}\right)\right|$ and $\mathrm{d} D P(\theta) \cdot \mathrm{d} D P\left(\theta_{j}\right)<0$. If so, major peak at $P_{j}$ (circle in Figure $7 \mathrm{~b}$ ) and its adjacent point $P_{j-1}$ or $P_{j+1}$ (square in Figure $7 \mathrm{~b}$ ) indicate a pair of P-Breaks. Otherwise, each major peak in Figure 7a indicates a pending I-Break. The possible isolated I-Break should be supplemented by another break or directly removed to ensure an even number of final I-Breaks. After paired breaks are detected, the P-Mutation and I-Mutation are finally grouped and the Step 0 sequences can be obtained.

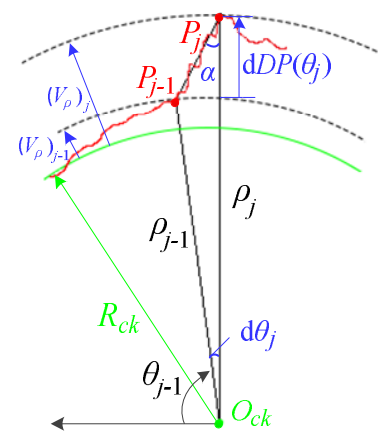

Figure 8. Break between point $P_{j-1}$ and $P_{j}$ in polar coordinate system, in which $O_{c k}$ denotes the pole, $\rho$ and $\theta$ denote polar coordinates, $R_{c k}$ is radius of the fitted circle, $V_{\rho}$ is radial distance between contour point and the fitted circle, $\mathrm{d} D P\left(\theta_{j}\right)$ and $\mathrm{d} \theta_{j}$ are radial and angular difference between the two points, and $\alpha$ is the angle between line $P_{j-1} P_{j}$ and radial direction at point $P_{j}$.

The LTBC algorithm is designed for $V_{\rho}$ correction to points in mutations according to the linear trends of adjacent $S_{t e p_{0}}$ sequences. In Figure 9, magenta and green solid lines denote detected I-Mutation to be corrected, and the blue lines denote the last and next $S t e p_{0}$ sequence. As shown in Figure 9, the $B_{1}, S t e p_{\mathrm{b}}$, and $B_{2}$ segments in a mutation are corrected separately. For an I-Mutation in small angular range, the last $S t e p_{0}$ and next $S t e p_{0}$ are fitted to line $f_{l}$ and $f_{n}$ respectively (red lines), and the short line $f_{\mathrm{c}}$ (red line) connecting $f_{l}$ to $f_{n}$ is used as a reference for Step $p_{\mathrm{b}}$ correction. $V_{\rho}$ of Step $\mathrm{b}$ segment is firstly corrected by translation, and the translation $t$ is calculated by least square estimation according to Equation (11):

$$
\left(c V_{\rho}\right)_{i}=\left(V_{\rho}\right)_{i}+t
$$

where $\left(V_{\rho}\right)_{i}$ denotes $V_{\rho}$ of each point in $S t e p_{\mathrm{b}}$ to be corrected and $\left(c V_{\rho}\right)_{i}$ denotes $V_{\rho}$ of each point in the referential $f_{\mathrm{c}}$ sequence. After $V_{\rho}$ of $S t e p_{\mathrm{b}}$ segment is corrected (green dashed line), the $V_{\rho}$ of $B_{1}$ segment is then corrected by linear transformation (magenta dashed line) based on Equation (12):

$$
\left(V_{\rho}\right)_{i}^{\prime}=V_{l E}+\frac{V_{b S}^{\prime}-V_{l E}}{V_{b S}-V_{l E}} \times\left(\left(V_{\rho}\right)_{i}-V_{l E}\right)
$$

where $\left(V_{\rho}\right)_{i}$ and $\left(V_{\rho}\right)_{i}^{\prime}$ denote $V_{\rho}$ of each point in $B_{1}$ segment before and after correction, $V_{l E}$ is $V_{\rho}$ of the ending point in last $S_{t e p_{0}}$, and $V_{b S}$ and $V_{b S}^{\prime}$ denote $V_{\rho}$ of the starting point in $S_{t e p_{\mathrm{b}}}$ before and after correction. This linear transformation method of $B_{1}$ correction ensures smooth transition from last Step 0 to corrected Step $\mathrm{b}$. Finally, $V_{\rho}$ of $B_{2}$ segment is corrected similarly to $B_{1}$ for smooth connection 
between corrected Step $\mathrm{b}_{\mathrm{b}}$ and the next Step $_{0}$. The LTBC method also applies to P-Break group with Step $\mathrm{b}_{\mathrm{b}}$ of one point.

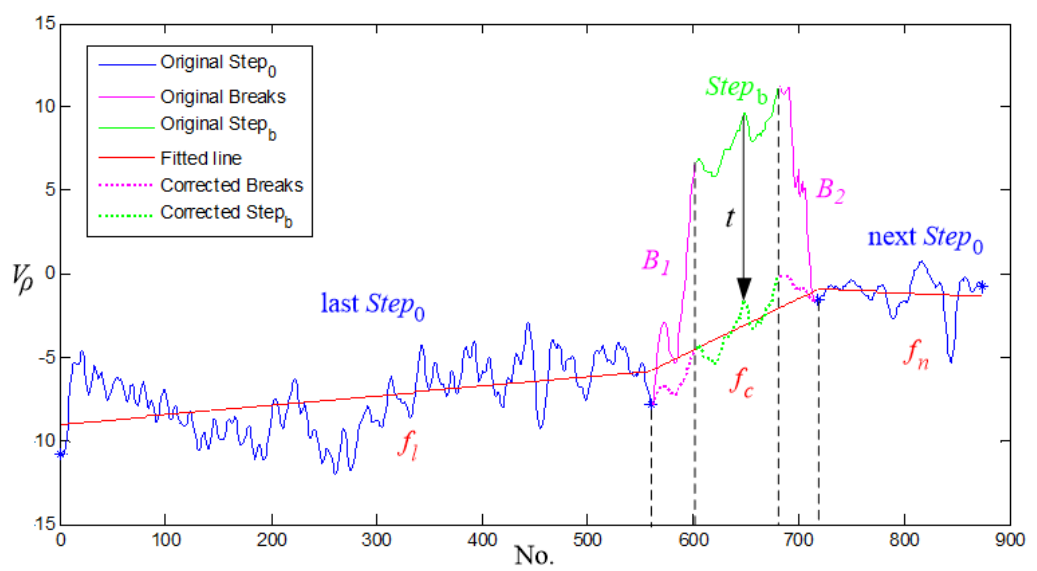

Figure 9. LTBC to $V_{\rho}$ of I-Mutation, in which vertical axis is radial distance to the fitted circle, and horizontal axis is the dynamic number rather than polar angle $\theta$, to avoid the discontinuity of clockwise $\theta$ ranging from $180^{\circ}$ to $-180^{\circ}$. The blue lines are $S t e p_{0}$, red lines are corresponding fitted lines, the magenta and green solid lines are detected I-Mutation, black arrow is the translation for Step $p_{\mathrm{b}}$ correction, and the magenta and green dashed lines are corrected sequence.

Repeat the above-mentioned steps of BDG, LTBC, and $V_{\rho}(\theta)$ sequence update until less than three break groups are detected. After correction to $V_{\rho}$, the corrected points are converted from polar coordinates $C_{k}\left(R_{c k}+V_{\rho}\left(\theta_{i}\right), \theta_{i}\right)$ to pixel coordinates $C_{k}\left(u_{i}, v_{i}\right)$.

\subsection{Cross-Section Dimension Measurement}

In our framework, a chessboard is placed near the power cable cross-section and the chessboard plane is coplanar with the power cable cross-section plane. Using the chessboard, the homography matrix mapping chessboard world coordinates of chessboard corners to the pixel coordinates can be estimated. According to Equation (1), mapping from spatial point $P$ on the chessboard plane to image point $p$ can be described by a 3-by-3 homography matrix $H$ as Equations (13) and (14):

$$
\begin{gathered}
\widetilde{p}=H \widetilde{P}, \\
H=\left[\begin{array}{lll}
H_{11} & H_{12} & H_{13} \\
H_{21} & H_{22} & H_{23} \\
H_{31} & H_{32} & H_{33}
\end{array}\right]=\lambda M\left[\begin{array}{lll}
r_{1} & r_{2} & T
\end{array}\right],
\end{gathered}
$$

where $\lambda$ is an arbitrary scale factor.

The homography matrix $H$ has only 8 free elements and can be normalized by $H_{33}=1$. For measurement image after undistortion, we can calculate $H$ with chessboard world coordinates $\left(X_{w}, Y_{w}\right)$ and pixel coordinates $(u, v)$ of chessboard corners according to Equation (15):

$$
\left[\begin{array}{cccccccc}
X_{w} & Y_{w} & 1 & 0 & 0 & 0 & -u X_{w} & -u Y_{w} \\
0 & 0 & 0 & X_{w} & Y_{w} & 1 & -v X_{w} & -v Y_{w}
\end{array}\right]\left[\begin{array}{l}
H_{11} \\
H_{12} \\
H_{13} \\
H_{21} \\
H_{22} \\
H_{23} \\
H_{31} \\
H_{32}
\end{array}\right]=\left[\begin{array}{c}
u \\
v
\end{array}\right] .
$$


Since the chessboard is coplanar with the power cable cross-section, the estimated homography matrix $H$ can also be used to map the pixel coordinates of cross-section contours to chessboard world coordinates. The chessboard world coordinates $C_{k}\left(X_{w}, Y_{w}\right)$ of contour points can be calculated with pixel coordinates $C_{k}(u, v)$ and $H$ according to Equation (16) after multilayer contour detection:

$$
\left[\begin{array}{cc}
H_{11}-u H_{31} & H_{12}-u H_{32} \\
H_{21}-v H_{31} & H_{22}-v H_{32}
\end{array}\right]\left[\begin{array}{c}
X_{w} \\
Y_{w}
\end{array}\right]=\left[\begin{array}{c}
u-H_{13} \\
v-H_{23}
\end{array}\right] .
$$

After $C_{k}\left(X_{w}, Y_{w}\right)$ is obtained, the diameter $D_{i}$ is measured based on Equation (17) of the perimeter tape method. As shown in Figure 10a, we first extract the convex hull [41] of the dense contour points in a layer, and the sum of convex vertex distance is used as perimeter $L$ to simulate the manual measurement of the perimeter tape:

$$
D_{i}=L_{i} / \pi, i=1,5,6,7,8 \text {. }
$$

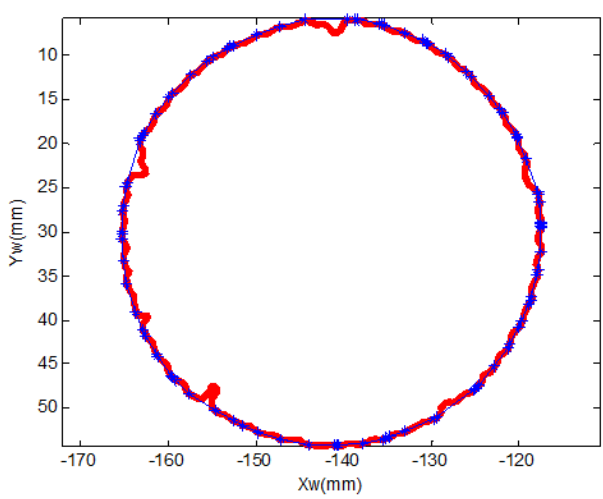

(a)

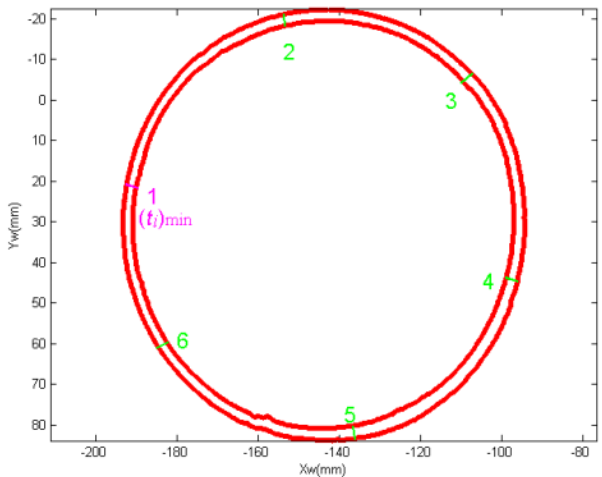

(b)

Figure 10. Cross-section measurement. (a) Perimeter measurement, in which red dots are contour $C_{k}$ and blue asterisks are vertexes of convex hull; (b) Thickness measurement, in which magenta line is minimum thickness and green lines are the other five thickness measurements clockwise.

To measure the thickness of layer $i(i=3,4,5,7,8)$, each point $P_{(i-, j)}$ of its inner contour is traversed to calculate the thickness $t_{(i-, j)}$ at the $j$-th point. As shown in Figure 10b, the minimum thickness $\left(t_{i}\right)_{\min }$ of layer $i$ is the minimum of all $t_{(i-, j)}$, and the mean thickness $\bar{t}_{i}$ is the mean value of six $t_{(i-, j)}$ measured at angle interval of 60 degrees from $\left(t_{i}\right)_{\min }$.

The eccentricity of insulation layer is defined as Equation (18):

$$
e_{4}=\left(t_{4 \max }-t_{4 \min }\right) / t_{4 \max }
$$

where $t_{4 \min }$ and $t_{4 \max }$ are the minimum and maximum thickness of insulation layer respectively.

\section{Results and Discussion}

\subsection{Cross-Section Measurement of Simulated Cable}

A chessboard containing $7 \times 6$ squares is designed in AutoCAD and the side length of each square is $10 \mathrm{~mm}$. In order to verify the effectiveness of the proposed method, we firstly simulate a cable cross-section with concentric circles in AutoCAD, in which wrapped tape (layer 2 ) is exaggeratedly drawn. The simulated pattern and chessboard are printed on an A4 paper, and this printed paper is captured by a CMOS camera (Nikon, Tokyo, Japan) with image resolution of $6000 \times 4000$ as simulated image $I_{1}$ (Figure 11a). Gaussian noise with variance of 0.01 and salt and pepper noise with density of 
0.01 are successively added to the $R, G$ and $B$ channels of $I_{1}$, and then we can get the simulated image $I_{2}$ shown in Figure 11b.

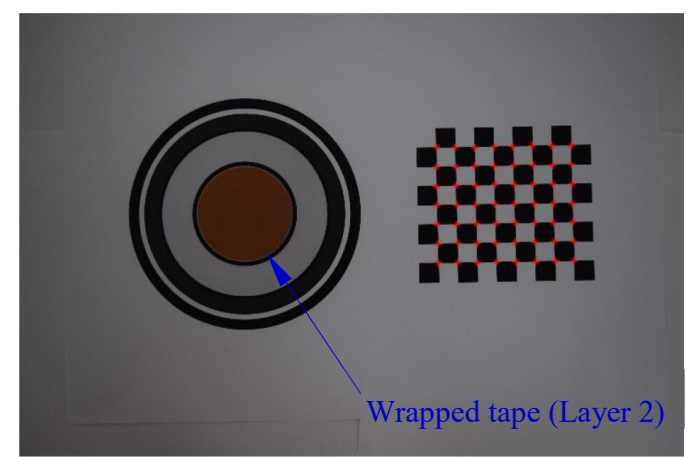

(a)

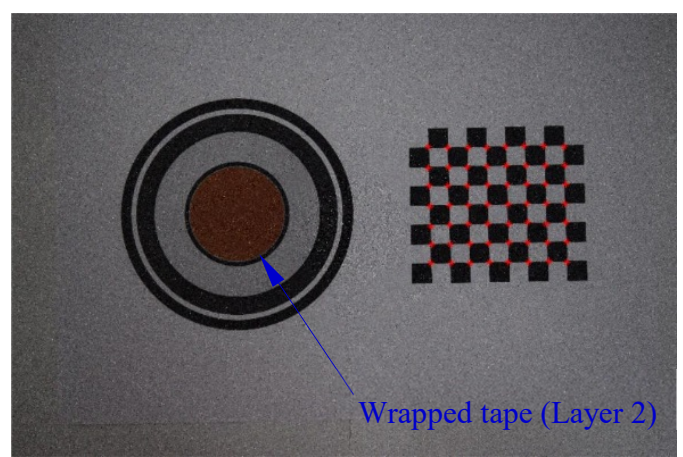

(b)

Figure 11. Simulated images. (a) Original image $I_{1} ;$ (b) Image $I_{2}$ with additive Gaussian noise and salt and pepper noise.

\subsubsection{Multilayer Contours Detection}

Coarse contour of copper conductor is firstly extracted based on chrominance information, and those of aluminum sheath are then obtained from grayscale image based on brightness information. The candidate edges are obtained by Canny detection and result of $I_{2}$ with mixed noise is shown in Figure 12a. In Canny edge detection, the standard deviation of Gaussian filter is set to $\sqrt{2}$ for noise reduction, the high threshold $t_{\text {high }}$ is dynamically 70 th percentile of gradients of whole image, and the low threshold is $0.4 t_{h i g h}$. The proportional threshold $t_{l}$ is set to 0.25 for long structural edges, and $t_{s}$ is set to 64 pixels for very short interference details. Since long structural edges with $e^{\prime}$ more than 0.9 are close to lines, the threshold $e_{\min }^{\prime}$ for short structural edges is loosely set to the minimum $e^{\prime}$ of these long linear edges. The whole structural edges of $I_{1}$ and $I_{2}$ are obtained by filtering with length and linearity features, and the result of $I_{2}$ is shown in Figure 12b.

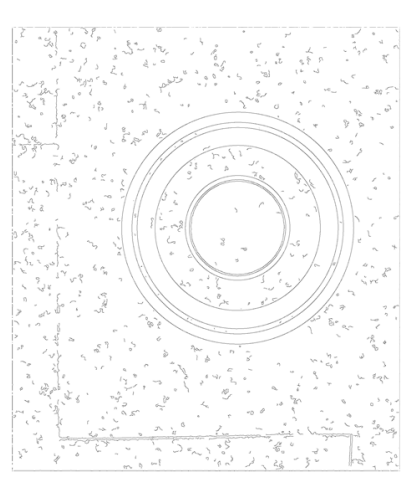

(a)

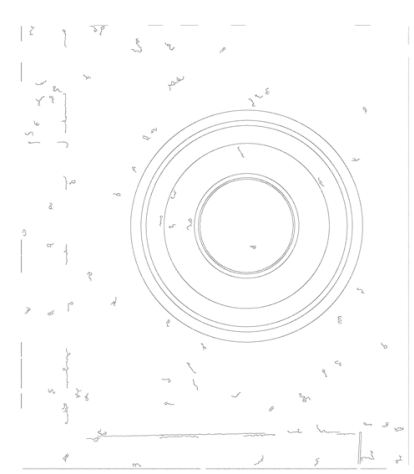

(b)

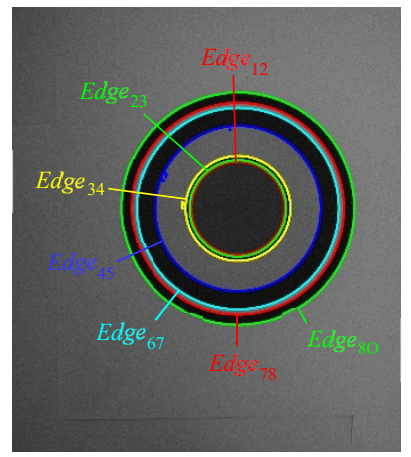

(c)

Figure 12. Edge detection and layering of $I_{2}$ with mixed noise. (a) Candidate edges by Canny detection; (b) Structural edges by filtering based on length and linearity; (c) Layering result of $I_{2}$, in which edges in different layers are rendered with different colors.

For edge layering, $\operatorname{Edge}_{k}(k=12,67,78,80)$ is firstly extracted from structural edges based on coarse contours, and $\operatorname{Edge}_{k}(k=23,34,45)$ is then extracted by annulus division. As shown in Figure $12 c$, nearly all edges (including $E d g e_{23}$ ) can be extracted from $I_{2}$ except that $E d g e_{56}$ in $I_{2}$ is covered by the mixed noise. Discontinuous structural edges in $I_{1}$ and $I_{2}$ are then connected by arc-based method with search radius of 1 . As shown in Figure 13, there are several edge discontinuities in $E d g e_{12}$ (yellow dots) in $I_{1}$ and $I_{2}$, which are connected with magenta dots and enclosed in this step. Since $E d g e_{56}$ between insulation screen (layer 5) and wrapped buffer (layer 6) with close grayscale in 
$I_{2}$ is missing after edge layering, we try to detect the $E d g e_{56}$ and $C_{56}$ from enhanced image. As shown in Figure 14a, grayscale $[0,0.2]$ in the original grayscale image of $I_{2}$ is mapped to $[0,1], E d g e_{56}$ (yellow dots) is then detected in the enhanced grayscale image, and closed contour $C_{56}$ can be obtained by arc-based edge connection (magenta dots). The arc-based edge connection relies on the previously detected edge, so connection result would absorb the deviations of inaccurate edge (yellow dots in red circle in Figure 14b) caused by busy background.

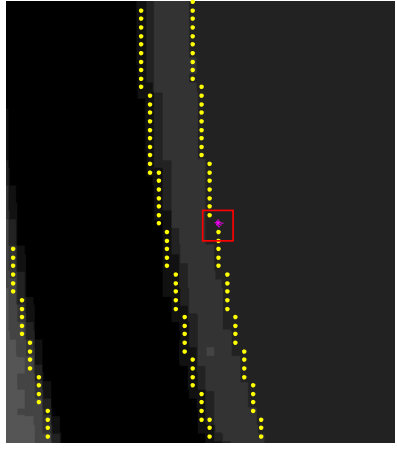

(a)

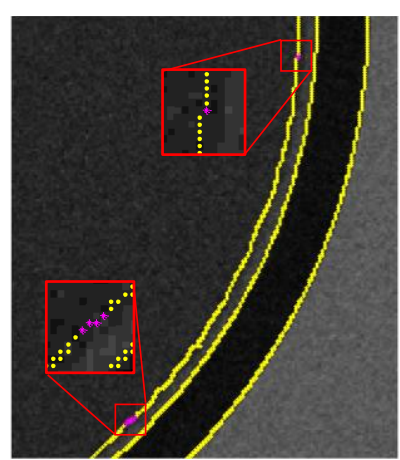

(b)

Figure 13. Arc-based edge connection, in which yellow dots are previous edges and magenta dots are arc-based connections. (a) Edge connection of $I_{1} ;(\mathbf{b})$ Edge connection of $I_{2}$.

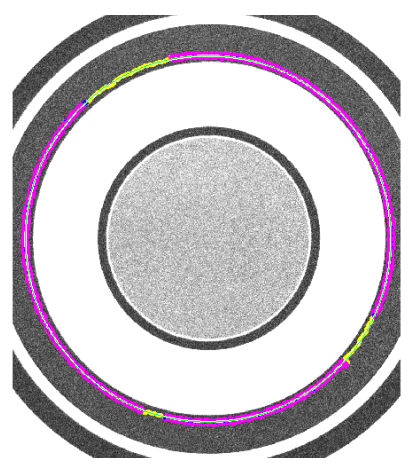

(a)

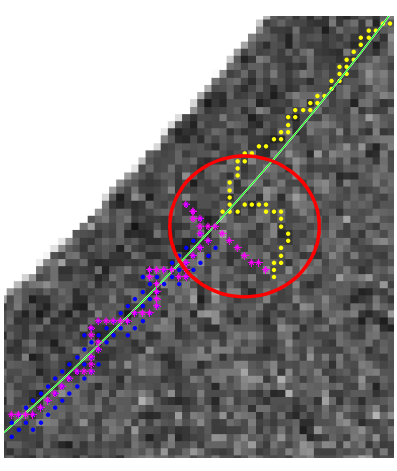

(b)

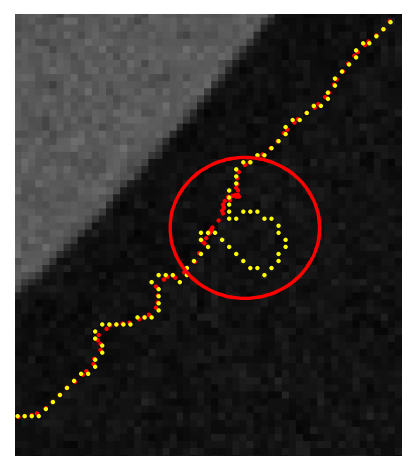

(c)

Figure 14. Edge $e_{56}$ connection and $C_{56}$ refinement. (a) Arc-based connection of $E d g e_{56}$ with the enhanced grayscale image as base map, in which yellow dots are $E d g e_{56}$, blue dots denote search range and magenta asterisks are accepted search points; (b) Partial enlargement of (a); (c) LTBC to a mutation in edge connection result, in which yellow dots are initial closed $C_{56}$ and red dots are refined results after corrections to detected mutation.

Each initial $C_{k}$ is fitted to circular contours $C_{c k}$ with max fitting error of $\sigma_{k}$. Major peaks with corresponding breaks are extracted by thresholds of $t_{\rho}=0.9 \sigma_{k}$ and $t_{\alpha}=45$, and $t_{p}$ is set to 0.7 for paired P-Breaks determination in adjacent location of major peaks. As shown in Figure 14c, one P-Mutation in initial $C_{56}$ (yellow dots in red circle) of $I_{2}$ is detected by BDG, LTBC is then made to the mutation, and the refined results are plotted with red dots, which reduces the bias to some extent.

\subsubsection{Cross-Section Dimension Measurement}

Extracted contour points are drawn with red dots in Figure 15, and their vertexes of convex hull are plotted with blue asterisks. Figure 15 shows that all contours required (including exaggeratedly drawn $C_{23}$ ) can be extracted from simulated images. Locations of minimum thickness $\left(t_{i}\right)_{\min }$ are drawn with magenta lines in Figure 15, and the other five thicknesses measured clockwise are drawn with green lines. Influenced by noise, $\left(t_{i}\right)_{\min }$ locations in $I_{1}$ and $I_{2}$ may be inconsistent, so the six thicknesses measured from $\left(t_{i}\right)_{\min }$ location for mean thickness calculation are all different. 


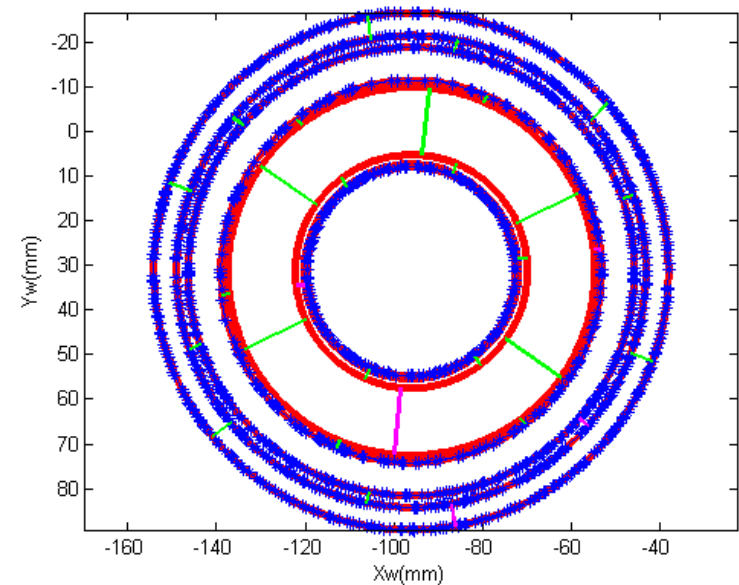

(a)

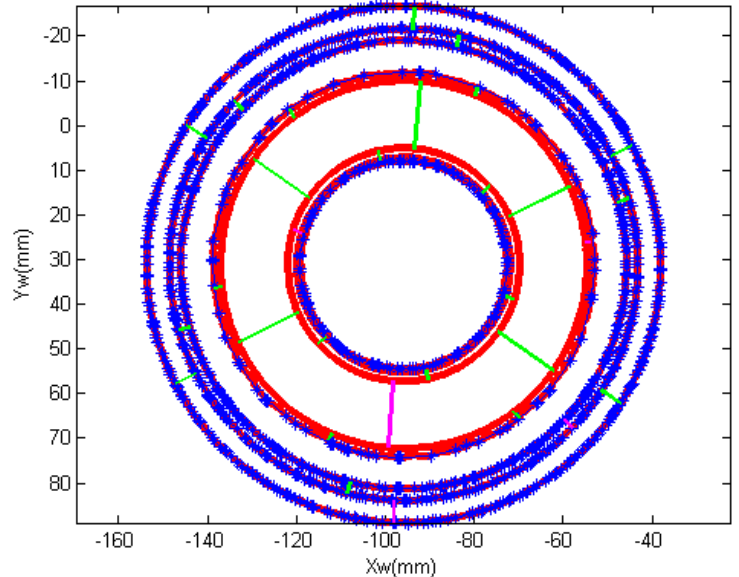

(b)

Figure 15. General view of cross-section measurement. (a) Perimeter and thickness for $I_{1}$, in which red dots are extracted contour points, blue asterisks are vertexes of contour convex hull, magenta lines denote minimum thickness $\left(t_{i}\right)_{\min ^{\prime}}$ and green lines are the other five thickness measurements clockwise; (b) Perimeter and thickness for $I_{2}$.

The diameter measurement results are listed in Table 1 , in which $D_{1}$ and $D_{2}$ are diameter measurements of $I_{1}$ and $I_{2}$, and $\left|V_{D 1}\right|$ and $\left|V_{D 2}\right|$ are corresponding errors between vision-based measurements and manual measurements $D_{0}$. Diameters are measured with RMSE of $0.424 \mathrm{~mm}$ for image $I_{1}$, and the mixed noise brings about a slight increase in diameter measurement errors for image $I_{2}$ with RMSE of $0.502 \mathrm{~mm}$. Compared with $I_{1}$, the salt and pepper noise will cover the contour $C_{56}$ to some extent and leading to a larger diameter measurement error for layer 5 in $I_{2}$. Since the RMSE of image $I_{1}$ and $I_{2}$ is almost equal, the diameter measurement is robust to noise.

Table 1. Diameter measurement results for $I_{1}$ and $I_{2}$. (unit: $\mathrm{mm}$ ).

\begin{tabular}{cccccc}
\hline Layer & $\boldsymbol{D}_{0}$ & $\boldsymbol{D}_{1}$ & $\left|\boldsymbol{V}_{\boldsymbol{D} 1}\right|$ & $\boldsymbol{D}_{2}$ & $\left|\boldsymbol{V}_{\boldsymbol{D} 2}\right|$ \\
\hline 8 & 115.470 & 115.958 & 0.488 & 115.772 & 0.302 \\
7 & 105.600 & 105.694 & 0.094 & 105.499 & 0.101 \\
6 & 99.910 & 100.557 & 0.647 & 100.390 & 0.480 \\
5 & 85.330 & 85.791 & 0.461 & 86.292 & 0.962 \\
1 & 46.752 & 46.901 & 0.149 & 46.681 & 0.071 \\
RMSE & - & - & 0.424 & - & 0.502 \\
\hline
\end{tabular}

The thickness measurement results are listed in Table 2, in which $t_{\min 1}, \bar{t}_{1}, t_{\min 2}$ and $\bar{t}_{2}$ are thickness measurements for $I_{1}$ and $I_{2}$ respectively. $t_{\min }$ and $\bar{t}$ in a layer are theoretically equal for concentric circles, while it's not true in manual and vision-based measurements due to errors caused by printing, imaging, and noise. Mean thickness for $I_{1}$ and $I_{2}$ are measured with RMSE of $0.063 \mathrm{~mm}$ and $0.058 \mathrm{~mm}$, which reflects to some extent the overall measurement accuracy, and minimum thickness measurement errors are with RMSE of $0.103 \mathrm{~mm}$ and $0.243 \mathrm{~mm}$, which shows the accuracy in worst cases. The mixed noise in image $I_{2}$ may cover local edges, so the minimum thicknesses especially that in layer 5 are measured with a relatively larger RMSE. The insulation eccentricity is theoretically 0 and the manual measurement is 0.013 . Influenced by both the measurement errors of maximum and minimum thickness, the $e_{4}$ measurements in Table 3 are 0.015 for $I_{1}$ and 0.014 for $I_{2}$ with very small measurement errors. 
Table 2. Thickness measurement results for $I_{1}$ and $I_{2}$. (unit: mm).

\begin{tabular}{|c|c|c|c|c|c|c|c|c|c|c|}
\hline \multirow{2}{*}{ Layer } & \multicolumn{2}{|c|}{ Manual } & \multicolumn{4}{|c|}{$I_{1}$} & \multicolumn{4}{|c|}{$I_{2}$} \\
\hline & $t_{\min 0}$ & $\bar{t}_{0}$ & $t_{\min 1}$ & $\left|V_{t \mathrm{~m} 1}\right|$ & $\bar{t}_{1}$ & $\left|V_{\bar{t} 1}\right|$ & $t_{\min 2}$ & $\left|V_{t \mathrm{~m} 2}\right|$ & $\bar{t}_{2}$ & $\left|V_{\bar{t} 2}\right|$ \\
\hline 8 & 4.820 & 4.995 & 4.941 & 0.121 & 5.051 & 0.099 & 4.936 & 0.116 & 5.074 & 0.079 \\
\hline 7 & 2.450 & 2.552 & 2.446 & 0.004 & 2.476 & 0.048 & 2.411 & 0.039 & 2.516 & 0.036 \\
\hline 5 & 1.520 & 1.552 & 1.328 & 0.192 & 1.510 & 0.073 & 0.999 & 0.521 & 1.646 & 0.094 \\
\hline 4 & 14.980 & 15.045 & 14.944 & 0.036 & 14.896 & 0.036 & 14.894 & 0.086 & 15.041 & 0.004 \\
\hline 3 & 1.900 & 2.020 & 1.891 & 0.009 & 2.045 & 0.032 & 1.861 & 0.039 & 2.033 & 0.013 \\
\hline RMSE & - & - & - & 0.103 & & 0.063 & & 0.243 & & 0.058 \\
\hline
\end{tabular}

Table 3. Eccentricity measurement of insulation layer in $I_{1}$ and $I_{2}$.

\begin{tabular}{cccc}
\hline Method & $\boldsymbol{t}_{4 \max }(\mathbf{m m})$ & $\boldsymbol{t}_{4 \min }(\mathbf{m m})$ & Insulation Eccentricity $\boldsymbol{e}_{4}$ \\
\hline Manual & 15.180 & 14.980 & 0.013 \\
$I_{1}$ & 15.171 & 14.944 & 0.015 \\
$I_{2}$ & 15.106 & 14.894 & 0.014 \\
\hline
\end{tabular}

\subsection{Cross-Section Measurement of Actual Cable}

The chessboard pattern is printed on an A4 image and used for homography matrix estimation. Actual cable specimen is cut with an axial length of about $100 \mathrm{~mm}$, cable cross-section and chessboard are simply pressed by the glass for coplanar condition, and the cross-section image is captured and shown in Figure 16. Note that the wrapped semi-conductive tape (Layer 2) is invisible to naked eyes in this cross-section image. All parameters in multilayer contour detection of actual specimen image are selected in the same way as those of simulated images above.

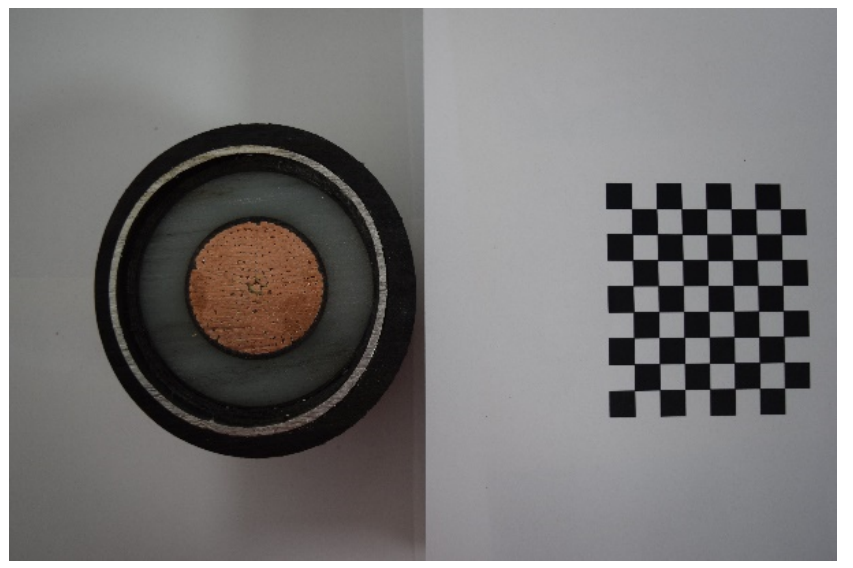

Figure 16. Image of actual specimen cross-section.

\subsubsection{Multilayer Contours Detection}

Linear combination $U^{\prime}$ is firstly constructed to express the yellow component offset from brightness, and coarse contour of copper conductor is segmented from $U^{\prime}$. Let $U_{\max }=1$ to enhance the $U^{\prime}$ perception of color difference, and Equation (7) can be simplified as $U^{\prime}=\alpha R+(1-\alpha) G-B$. Considering the color of copper conductor approximates $R G B(173,113,68)$, the linear combination $U^{\prime}=0.6 R+0.4 G-B$ is constructed with $\alpha:(1-\alpha)=173: 113$. As shown in Figure 17a, coarse contour $r C_{12}$ conforms well to the actual edges of copper conductor except for a few deviations caused by specular reflection point on contour segmented to the background. Coarse contours of aluminum sheath (layer 7) are then segmented from grayscale image and $r C_{67}$ (blue dots) and $r C_{78}$ (green dots) are plotted in Figure 17b. To illustrate the details of coarse aluminum contours, partial enlargement of Figure $17 \mathrm{~b}$ is drawn in Figure 17c, from which we can see $r C_{78}$ and $r C_{67}$ conform well to actual 
contours except for a few deviations in $r C_{67}$ (blue dots in magenta ellipses) caused by wrapped buffer (layer 6) peeling off and aluminum sheath cutting burr.

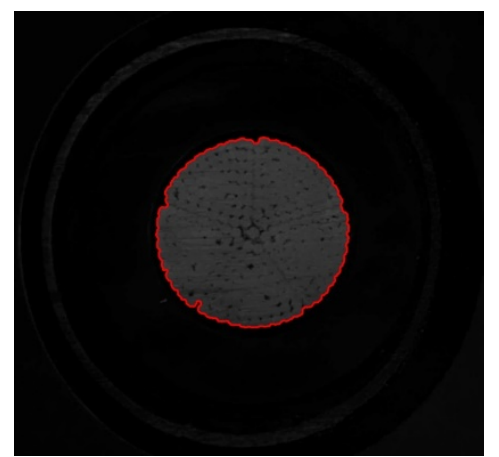

(a)

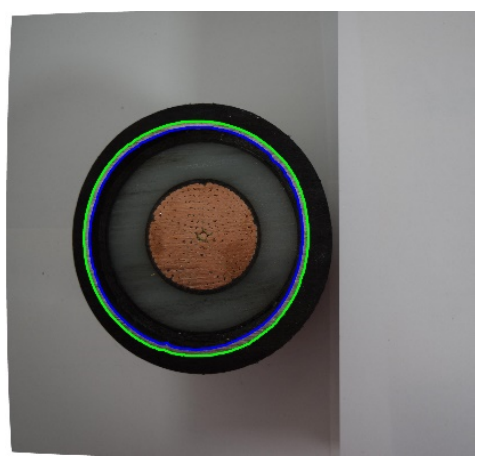

(b)

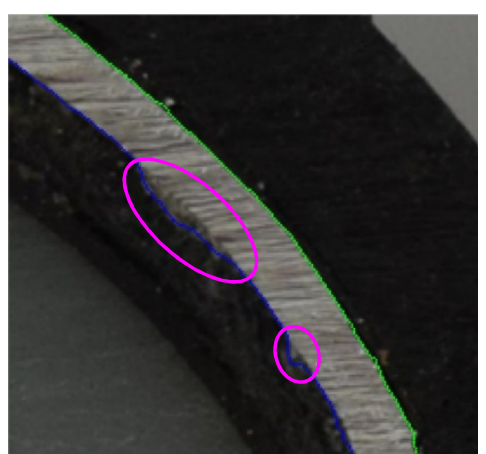

(c)

Figure 17. Coarse contour extraction of metal layers. (a) Extracted $r C_{12}$ plotted with red dots on $U^{\prime}$ component; (b) Extracted $r C_{67}$ in blue dots and $r C_{78}$ in green dots; (c) Partial enlargement of (b), in which deviations are circled.

The candidate edges are obtained by Canny detection and shown in Figure 18a, including structural edge, details inside each layer, stain and cutting texture. Thresholds for filtering based on length and linearity features are selected in the same way as those of simulated images. After edge filtering, long edges and short edges with good linearity are reserved, and the whole structural edges are shown in Figure 18b.

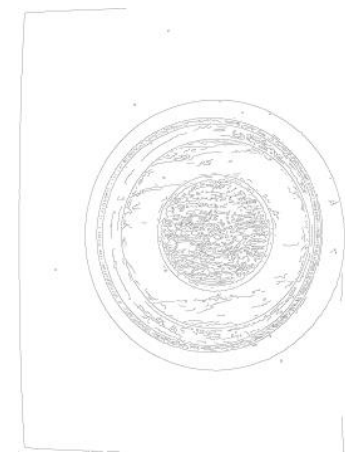

(a)

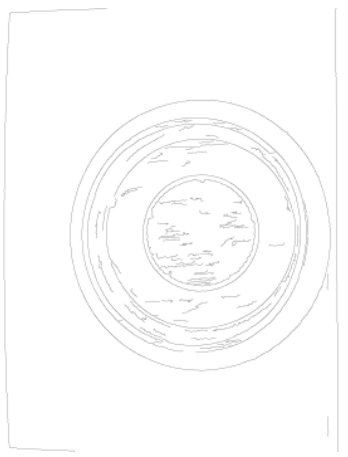

(b)

Figure 18. Edge detection. (a) Candidate edges by Canny detection. (b) Structural edges by filtering based on length and linearity features.

For edge points layering, cable region is firstly obtained by hole filling of structural edges, and the outermost boundary is extracted as Edge 80 and plotted in Figure 19a. Structural edges that are near the previously extracted coarse contours are then extracted as $E d g e_{k}(k=12,67,78)$ and drawn in Figure 19a. For the left structural edges related to layers with good concentric shapes, edge points are divided into different annuli and rendered with different colors (Figure 19b), and the annuli with structural edges of $E d g e_{34}$ and $E d g e_{45}$ are detected (Figure 19c) based on the point count and polar angle span. Since insulation screen (layer 5) and wrapped buffer (layer 6) are in close grayscale and location, few edge points of the contour between them exist in previously detected structural edges, and $E d g e_{56}$ cannot be extracted by annulus division. All layered edge points $E d g e_{k}(k=12,34,45,67,78,80)$ are plotted in Figure 20a. To illustrate the details of result, partial edges in Figure 20a are redrawn with red dots in Figure 20b, and the green lines denote the fitted circular contour of each layer. The distances between layered edges and fitted circles confirm the good concentric shape of inner layers and larger 
deformation of outer layers. Besides, it can be seen from Figure 20b that interference edge points (dots in blue ellipses) and discontinuity (dots in magenta ellipses) exist in the edge layering result.

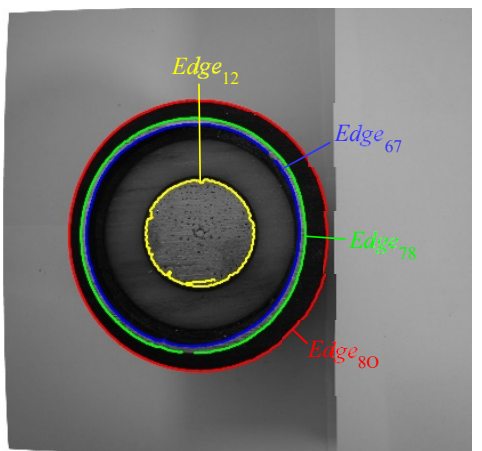

(a)

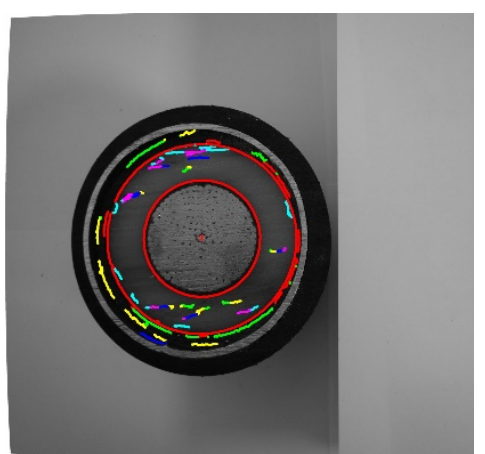

(b)

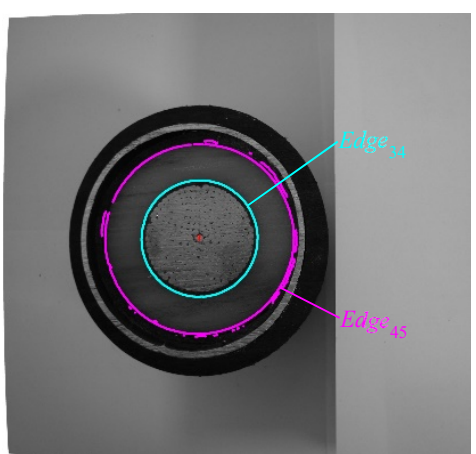

(c)

Figure 19. Edge layering. (a) Extraction based on previous coarse contours, in which edges in different layers are rendered with different colors; (b) Annulus division of the left structural edges, in which different annuli are rendered with different colors; (c) Annuli with structural edges.

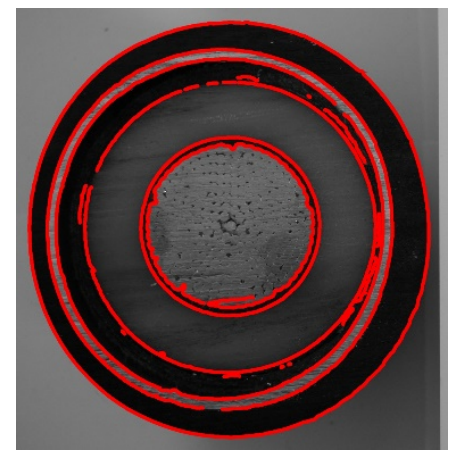

(a)

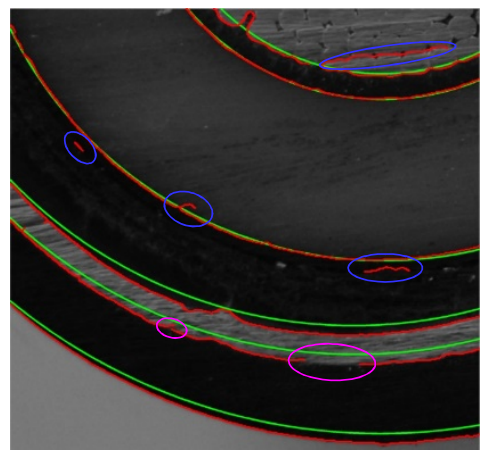

(b)

Figure 20. Layering result of edge points. (a) Layered edges plotted in red dots; (b) Partial enlargement of (a), in which red dots are layered edges, green lines are the fitted circular contour, and interferences and discontinuity exist in blue and magenta ellipses.

After layered $E d g e_{k}$ is obtained, discontinuous edges in each layer are connected by arc-based edge connection and the connections are shown in Figure 21a, in which yellow dots are previous $E d g e_{k}$ and magenta dots are connections. For this specimen with average stain, the discontinuities between arcs in extracted $E d g e_{k}$ are not long, so direct connection can meet the requirements. The only one closed contour in each layer after connection is the initial contour $C_{k}$, and the result without $C_{56}$ is shown in Figure 21b. The contrast of Figures 20a and $21 \mathrm{~b}$ shows that all unconnected structural edges are successfully connected, and the unconnected interferences are removed. Since Edge 56 is not extracted in previous edge layering, we try to detect $E d g e_{56}$ in enhanced grayscale image and closed $C_{56}$ is then obtained by edge connection of $E_{d g e_{56}}$ (Figure 22a). To illustrate the details of edge connection, three local positions in (Figure 22a is enlarged and shown in Figure 22b-d. The arc-based edge connection relies on the previously layered edges, connection result is reliable if Edge 56 is accurate (Figure 22b), and the result would absorb the deviations of inaccurate Edge 56 caused by busy background (Figure 22c,d). As shown in Figure 22c,d, there are several textures inside the wrapped buffer (layer 6) mistaken for

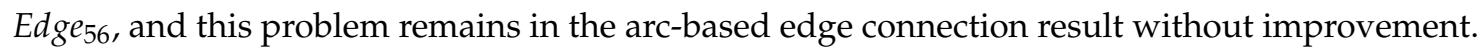




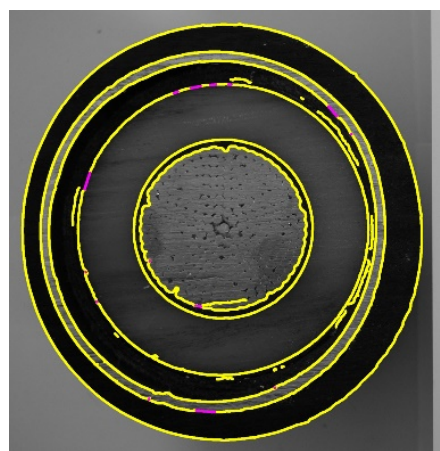

(a)

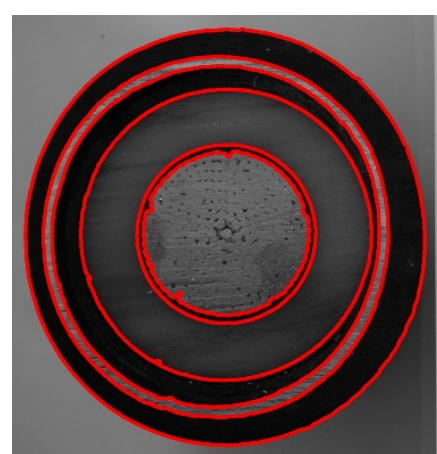

(b)

Figure 21. Arc-based edge connection result. (a) All connection locations, in which yellow dots are previous result and magenta dots are connections; (b) Closed initial $C_{k}$ after arc connection.

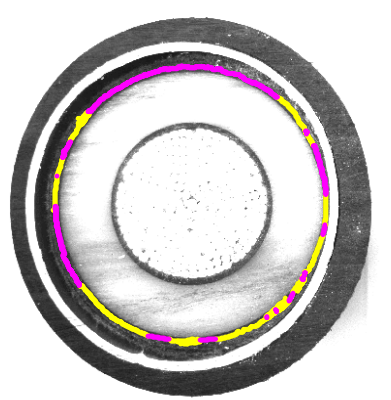

(a)

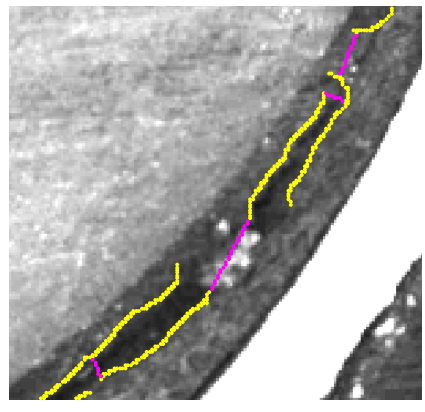

(c)

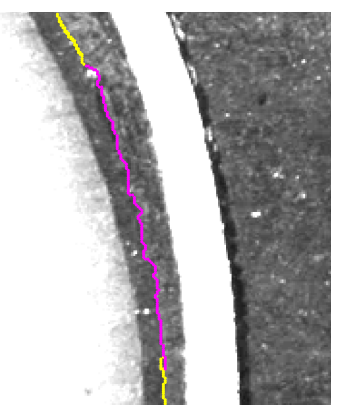

(b)
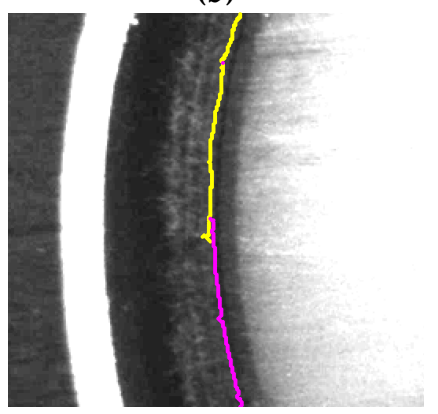

(d)

Figure 22. Edge 56 connection plotted on enhanced image. (a) Edge 56 connection, in which yellow dots are previous $E_{g} g_{56}$ and magenta dots are connections; (b) Partial enlargement of (a) with accurate connections; (c,d) are partial enlargements of (a) with inaccurate Edge 56 in yellow.

For contour refinement, one P-Mutation in initial $C_{45}$ and two P-Mutations in initial $C_{56}$ are detected by BDG algorithm and LTBC is made to these mutations. As shown in Figure 23a, yellow dots denote initial contour $C_{45}$, red dots are refined contour after LTBC, and the inaccurate edges caused by cutting texture of XLPE insulation are detected as mutation and corrected. As shown in Figure 23b, yellow dots denote initial contour $C_{56}$ and the steep edges in busy background are detected as mutation and corrected. The inaccurate edges of $C_{56}$ are caused by inaccurate arc-based edge connection in busy background (Figure 22c,d), LTBC algorithm can fix mutations in Figure 22c to Figure 23b with circle characteristics and sequence continuity, but inaccurate edges with gentle changes in radial direction (Figure 22d) are failed to be corrected. For other layers without break detected, it means good consistency of points in the contour. 


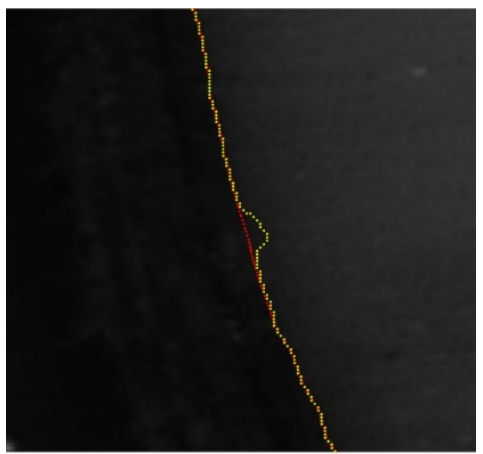

(a)

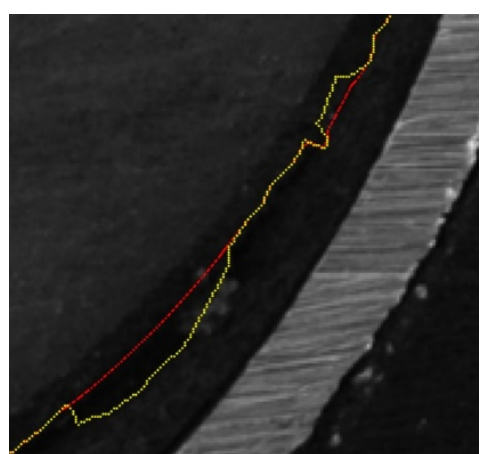

(b)

Figure 23. LTBC to detected mutations, in which yellow dots are initial closed contours and red dots are corrections. (a) LTBC to a detected P-Mutation in $C_{45}$; (b) LTBC to detected P-Mutations in $C_{56}$.

\subsubsection{Cross-Section Dimension Measurement}

Extracted contour points are drawn with red dots in Figure 24, and vertexes of their convex hull are plotted with blue asterisks. Similar to cross-section measurement of simulated images, it can be seen from Figure 24 that all contours required can be detected by this method except for invisible $C_{23}$. Table 4 is the measurement results of actual specimen cross-section. The $D_{0}, t_{\min 0}$ and $\bar{t}_{0}$ are manually measured diameter, minimum thickness and mean thickness, and the $D, t_{\min }$ and $\bar{t}$ are vision-based measurement results.

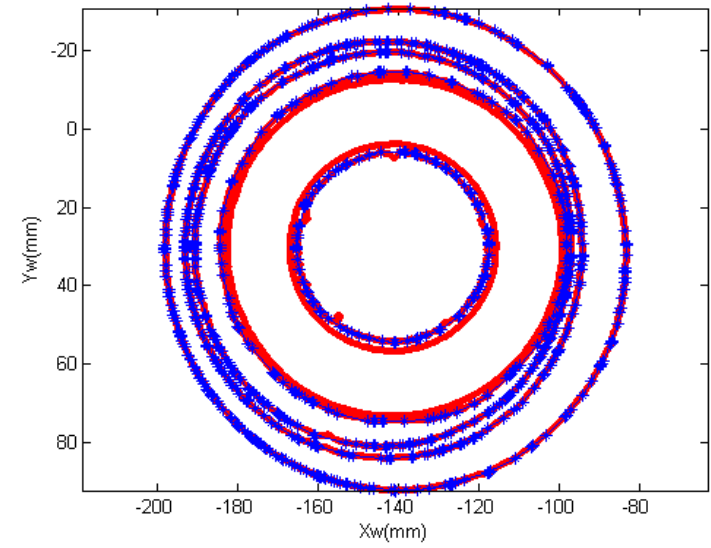

(a)

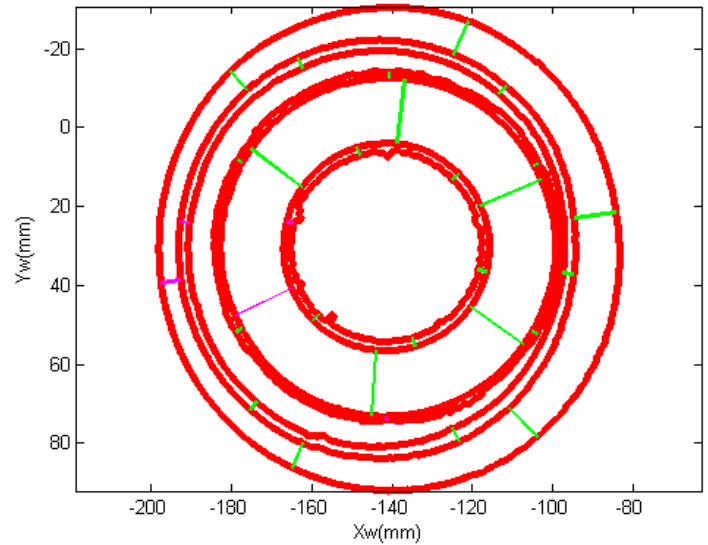

(b)

Figure 24. General view of cross-section measurement. (a) Perimeter measurement, in which red dots are extracted contour and blue asterisks are vertexes of contour convex hull; (b) Thickness measurement, in which magenta line denotes $\left(t_{i}\right)_{\min }$ and green lines are the other five measurements clockwise.

Table 4. Measurement results of actual specimen cross-section. (unit: mm).

\begin{tabular}{cccccccccc}
\hline Layer & $\boldsymbol{D}_{0}$ & $\boldsymbol{D}$ & $\left|\boldsymbol{V}_{\boldsymbol{D}}\right|$ & $\boldsymbol{t}_{\min 0}$ & $\boldsymbol{t}_{\min }$ & $\left|\boldsymbol{V}_{\boldsymbol{t} \min }\right|$ & $\overline{\boldsymbol{t}}_{0}$ & $\overline{\boldsymbol{t}}$ & $\left|\boldsymbol{V}_{\overline{\boldsymbol{t}}}\right|$ \\
\hline 8 & 118.729 & 119.207 & 0.478 & 4.940 & 4.839 & 0.101 & 7.628 & 7.903 & 0.275 \\
7 & 103.387 & 103.098 & 0.289 & 2.340 & 2.209 & 0.131 & 2.747 & 2.798 & 0.051 \\
6 & 96.784 & 97.580 & 0.796 & - & - & - & - & - & - \\
5 & 86.803 & 87.984 & 1.181 & 0.930 & 0.803 & 0.127 & 1.062 & 1.303 & 0.241 \\
4 & - & - & - & 16.140 & 15.438 & 0.702 & 16.325 & 16.175 & 0.150 \\
3 & - & - & - & 1.440 & 1.477 & 0.037 & 1.567 & 2.042 & 0.475 \\
1 & 48.000 & 48.176 & 0.176 & - & - & - & - & - & - \\
RMSE & - & - & 0.689 & - & - & 0.328 & - & - & 0.281 \\
\hline
\end{tabular}

The terms $\left|\mathrm{V}_{D}\right|,\left|V_{t \min }\right|$, and $\left|V_{\bar{t}}\right|$ are the corresponding measurement errors between vision-based and manual methods. Note that the outermost $D_{0}$ is measured with perimeter tape, and $D_{0}$ of other 
layers is the mean of multiple diameters nondestructively measured by a vernier caliper. $t_{\min 0}$ and $\bar{t}_{0}$ are nondestructive measurements on the cable cross-section. In Table 4, the RMSE of diameter measurement is $0.689 \mathrm{~mm}$, and the diameter error of layer 5 reaches up to $1.181 \mathrm{~mm}$. Though closed $C_{56}$ can be detected from the enhanced image, there are still several textures inside layer 6 mistaken for $C_{56}$ (Figure 22d), which bring about the local expansion of $C_{56}$ and larger diameter and mean thickness measurements of layer 5. For minimum thickness measurement, vision-based measurement results are all smaller than manual measurement results except for layer 3, and this consistency may be because point traverse in vision-based measurement is more sensitive to minimum value determination than naked-eye observation. $C_{23}$ is invisible and not extracted from image of actual cable cross-section, so slightly larger minimum thickness and mean thickness of layer 3 in Table 4 are calculated based on contours $C_{13}$ and $C_{34}$. The minimum thickness measurement error of insulation layer reaches $0.702 \mathrm{~mm}$, and no clear influence factors for this relatively large error have been found. The larger mean thickness and diameter measurements of the outermost layer may be related to the local expansion of $C_{80}$ due to cutting burrs shown in Figure 25. A different minimum thickness location between vision-based and manual measurement means six thickness measurement locations for mean thickness calculation are all different, and the overall minimum and mean thickness errors are with RMSE of $0.328 \mathrm{~mm}$ and $0.281 \mathrm{~mm}$. As shown in Table 5, manually measured $e_{4}$ is 0.025 , and the vision-based measurement influenced by measurement errors of maximum and minimum thicknesses is 0.072 . Note that the error of maximum thickness measurement of insulation layer is only $0.071 \mathrm{~mm}$, and the large error of eccentricity is mainly determined by the above-mentioned large error of minimum thickness.

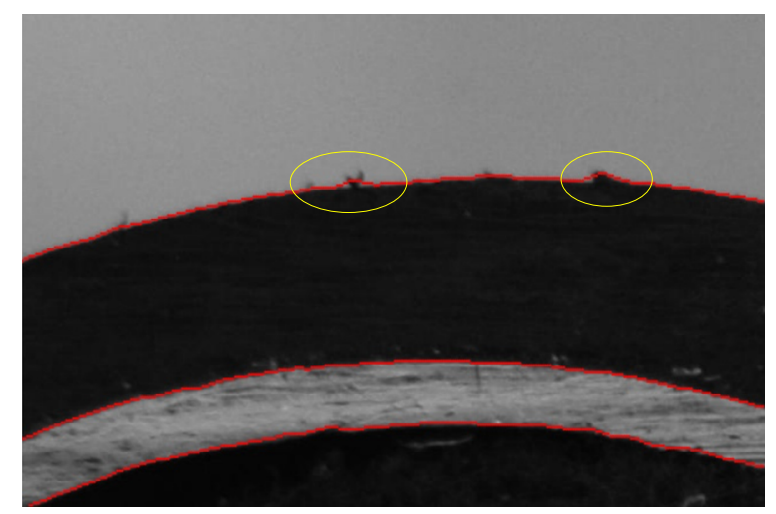

Figure 25. Cutting burrs of outermost contour.

Table 5. Eccentricity measurement of insulation layer.

\begin{tabular}{cccc}
\hline Method & $\boldsymbol{t}_{4 \max }(\mathbf{m m})$ & $\boldsymbol{t}_{4 \min }(\mathbf{m m})$ & Insulation Eccentricity $\boldsymbol{e}_{4}$ \\
\hline Manual & 16.560 & 16.140 & 0.025 \\
Image & 16.631 & 15.438 & 0.072 \\
\hline
\end{tabular}

In order to estimate the precision of this method, we performed four similar experiments with an actual specimen. In these experiments, specimen and chessboard are placed with different relative locations and rotations, and images are captured at different object distances. The terms $\left|\mathrm{V}_{D}\right|,\left|V_{t \min }\right|$, and $\left|V_{\bar{t}}\right|$ in Table 6 are the corresponding measurement errors between vision-based and manual methods in four experiments, and these statistical results are arranged for each measured item. Table 6 shows that diameters are measured with average RMSE of $0.768 \mathrm{~mm}$, and it reduces to $0.545 \mathrm{~mm}$ if we remove the measurements of layer 5 with inaccurate contour points. The minimum thicknesses are measured with average RMSE of $0.308 \mathrm{~mm}$, and the mean thicknesses are measured with average RMSE of $0.327 \mathrm{~mm}$. It can be seen from Table 7 that the insulation eccentricities in four experiments are measured with errors of $0.047,0.013,0.023$ and 0.022 , and the average error is 0.026 . 
Table 6. Measurement errors of diameters and thicknesses in four experiments. (unit: $\mathrm{mm}$ ).

\begin{tabular}{cccccccccc}
\hline Layer & $\mathbf{8}$ & $\mathbf{7}$ & $\mathbf{6}$ & $\mathbf{5}$ & $\mathbf{4}$ & $\mathbf{3}$ & $\mathbf{1}$ & RMSE & RMSE $_{\boldsymbol{a}}$ \\
\hline$\left|V_{D 1}\right|$ & 0.478 & 0.289 & 0.796 & 1.181 & - & - & 0.176 & 0.689 & \\
$\left|V_{D 2}\right|$ & 0.702 & 0.073 & 0.984 & 1.627 & - & - & 0.116 & 0.908 & 0.768 \\
$\left|V_{D 3}\right|$ & 0.568 & 0.192 & 0.876 & 1.132 & - & - & 0.056 & 0.694 & \\
$\left|V_{D 4}\right|$ & 0.611 & 0.145 & 0.893 & 1.362 & - & - & 0.078 & 0.781 & \\
$\left|V_{t \mathrm{~m} 1}\right|$ & 0.101 & 0.131 & - & 0.127 & 0.702 & 0.037 & - & 0.328 & \\
$\left|V_{t \mathrm{~m} 2}\right|$ & 0.428 & 0.038 & - & 0.113 & 0.343 & 0.070 & - & 0.253 & 0.308 \\
$\left|V_{t \mathrm{~m} 3}\right|$ & 0.434 & 0.028 & - & 0.110 & 0.518 & 0.170 & - & 0.316 & \\
$\left|V_{t \mathrm{~m} 4}\right|$ & 0.377 & 0.012 & - & 0.090 & 0.614 & 0.204 & - & 0.337 & \\
$\left|V_{\bar{t} 1}\right|$ & 0.275 & 0.051 & - & 0.241 & 0.183 & 0.475 & - & 0.281 & \\
$V_{\bar{t} 2} \mid$ & 0.326 & 0.118 & - & 0.413 & 0.219 & 0.475 & - & 0.336 & 0.327 \\
$V_{\bar{t} 3} \mid$ & 0.304 & 0.144 & - & 0.360 & 0.190 & 0.394 & - & 0.295 & \\
$\left|V_{\bar{t} 4}\right|$ & 0.289 & 0.144 & - & 0.607 & 0.245 & 0.507 & - & 0.397 & \\
\hline
\end{tabular}

Table 7. Measurement errors of insulation eccentricity in four experiments.

\begin{tabular}{cccccc}
\hline Experiment & $\boldsymbol{t}_{4 \max }(\mathbf{m m})$ & $\boldsymbol{t}_{4 \min }(\mathbf{m m})$ & Insulation Eccentricity $\boldsymbol{e}_{4}$ & Error & Error $_{\boldsymbol{a}}$ \\
\hline 1 & 16.631 & 15.438 & 0.072 & 0.047 & \\
2 & 16.429 & 15.797 & 0.038 & 0.013 & 0.026 \\
3 & 16.417 & 15.622 & 0.048 & 0.023 & \\
4 & 16.289 & 15.526 & 0.047 & 0.022 & \\
\hline
\end{tabular}

\subsection{Influence of Printed Chessboard on Measurement Accuracy}

The side length of each chessboard square is designed as $10 \mathrm{~mm}$ in AutoCAD, and the actual length in printed chessboard is on average $9.600 \mathrm{~mm}$ measured by a Vernier caliper. After image undistortion based on the actual size of chessboard, the homography matrix $H$ mapping the chessboard world coordinates of the chessboard corners to the pixel coordinates is calculated. To evaluate the influence of printed chessboard on plane measurement, the coordinates differences $\left(\mathrm{d} X_{w}, \mathrm{~d} Y_{w}\right)$ between mapped chessboard world coordinates $\left(X_{w}^{\prime}, Y_{w}^{\prime}\right)$ and actual coordinates $\left(X_{w}, Y_{w}\right)$ of chessboard corners are calculated. The coordinates differences in experiments of simulated cables and actual cables are shown in Figure 26a,b.

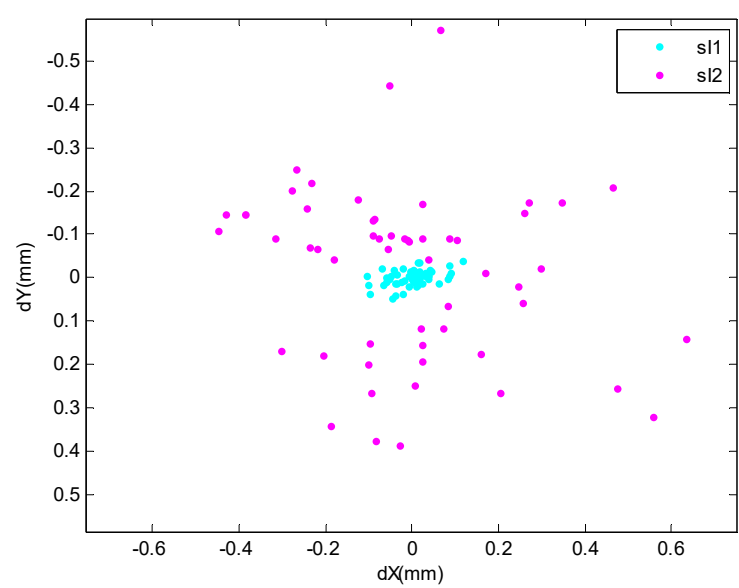

(a)

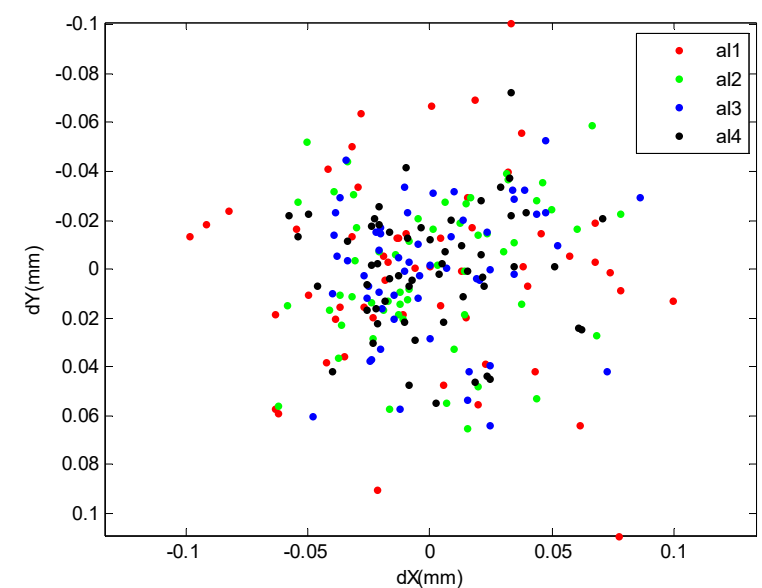

(b)

Figure 26. Coordinates differences between mapped and actual chessboard world coordinates of chessboard corners. (a) The coordinates differences in two experiments of simulated cable images; (b) The coordinates differences in four experiments of actual cable images. 
It can be seen from Figure 26 that most coordinates differences of chessboard corners in simulated cable images are within $0.05 \mathrm{~mm}$, and those in actual cable images are within $0.1 \mathrm{~mm}$. The placements of chessboard papers in experiments make a difference to chessboard smoothness and then corner point precision. Compared with cross-section measurement of simulated images, there is a loss of accuracy in the measurement results of actual cable images. In this homography matrix-based measurement method, the measurement accuracy is highly dependent on the coplanar precision of cable cross-section and the chessboard. For simulated images, cable cross-sections are strictly coplanar with the chessboard, so the homography matrix-based coordinate mapping is of higher positional accuracy. Since the actual specimen is simply pressed by the glass for coplanar condition, there are deviations between the mapped chessboard world coordinates and the physical positions of contour points, and the cross-section is measured with comparatively lower accuracy.

\section{Conclusions and Future Works}

To improve the geometric dimension measurement efficiency in power cable testing, a monocular vision-based cross-section measurement framework for single-core power cable was proposed in this paper. This vision-based framework consists of image undistortion, multilayer contour detection, and cross-section dimension measurement. The images of cable cross-section are firstly captured and undistorted with the camera calibration parameters. Then the contour of each layer is detected in the cable cross-section image. Finally, the diameter and thickness of different layers and eccentricity of insulation layer are calculated by the proposed homography matrix-based measurement method. The feasibility of this framework is verified by experiments on simulated images and actual images of power cable specimen cross-section. For multilayer contour detection, continuous contour of each layer can be synchronously extracted by edge detection and layering, arc-based edge connection, and contour refinement. And the proposed contour detection algorithm has good adaptability for structural deformation. Diameter, minimum thickness, mean thickness and insulation eccentricity of simulated image without additive noise are measured with RMSE of $0.424,0.103$ and $0.063 \mathrm{~mm}$, and 0.002 , respectively, and those of simulated image with mixed noised are measured with RMSE of $0.502,0.243$ and $0.058 \mathrm{~mm}$ and 0.001 . Diameter and mean thickness measurements are robust to noise, while the mixed noise will obviously increase the measurement error of minimum thickness due to local interferences. Diameter, minimum thickness and mean thickness of actual cable images are measured with averaged RMSE of $0.768,0.308$ and $0.327 \mathrm{~mm}$, and the insulation eccentricity result is most sensitive since it is influenced by both the maximum and minimum thickness in extreme cases.

There is a large potential for accuracy improvement in dimension measurement based on this monocular vision-based framework. In order to improve the measurement accuracy, the specimen should be first cleaned up before imaging to eliminate the influence of cutting burrs, debris, and stain. For the placement scheme of chessboard, high coplanar precision would contribute to accuracy improvement of this homography matrix-based cross-section measurement on one hand. On the other hand, a hollowed-out chessboard placed outside the cable cross-section would be considered to avoid the extrapolation from chessboard corners in coordinate mapping, however this puts forward a higher coplanar requirement for hardware design. As to contour detection algorithm, methods for accurate contour extraction from regions with close grayscale are needed.

Author Contributions: X.Z. proposed the method, designed the algorithm, performed the experiment and analysis, and wrote the manuscript. H.Y. supervised the research and revised the manuscript.

Funding: This work was funded by National Natural Science Foundation of China, grant number 51077105 and Science and Technology Research Program of the State Grid Corporation of China, grant number SGSX0000YJJS(2014)457.

Acknowledgments: We would like to express our gratitude to Chao Peng for providing power cable specimens and demonstrating traditional measurement methods.

Conflicts of Interest: The authors declare no conflict of interest. 


\section{References}

1. General administration of quality supervision, inspection and quarantine of the People's Republic of China. Standardization administration of the People's Republic of China. In Common Test Methods for Insulating and Sheathing Materials of Electric and Optical Cables_Part 11: Methods for General Application-Measurement of Thickness and Overall Dimensions_-Test for Determining the Mechanical Properties, 4th ed.; SAC: Beijing, China, 2008.

2. General administration of quality supervision, inspection and quarantine of the People's Republic of China. Standardization administration of the People's Republic of China. In Power Cables with Cross-Linked Polyethylene Insulation and Their Accessories for Rated Voltage of $110 \mathrm{kV}(\mathrm{Um}=126 \mathrm{kV})$, 3rd ed.; SAC: Beijing, China, 2014.

3. General administration of quality supervision, inspection and quarantine of the People's Republic of China. Standardization administration of the People's Republic of China. In Power Cables with Cross-Linked Polyethylene Insulation and Their Accessories for Rated Voltage of $220 \mathrm{kV}(\mathrm{Um}=252 \mathrm{kV}$ ), 2nd ed.; SAC: Beijing, China, 2015.

4. General administration of quality supervision, inspection and quarantine of the People's Republic of China. Standardization administration of the People's Republic of China. In Power Cables with Cross-Linked Polyethylene Insulation and Their Accessories for Rated Voltage of $500 \mathrm{kV}(\mathrm{Um}=550 \mathrm{kV})$, 1st ed.; SAC: Beijing, China, 2008.

5. Roller, R.; Ferber, R.; Loftus, W. Non-Destructive Eccentricity and Insulation Thickness Measurement system. U.S. Patent No. 3796874, 12 March 1974.

6. Salzmann, H.; Jung, U.; Kotter, W. Method and Apparatus for the Cross-Sectional Measurement of Electric Insulated Conductors. U.S. Patent No. 005976449, 2 November 1999.

7. Chen, J.; Li, B.; Wei, K.; Guo, L.; Qu, G. Development of online cable eccentricity detection system based on X-ray CCD. Nucl. Electron. Detect. Technol. 2008, 28, 462-465.

8. Feng, Y.; Xu, X.; Gong, J.; Liu, D. X-ray tube wall thickness eccentricity measurement platform. Sci. Technol. Innov. 2015, 2, 14-15.

9. Wang, L.; Wang, C.; Cheng, J.; Zhang, T.; Wang, H. Mathematical model establishment for cable eccentricity online detection system based on X-ray. In Proceedings of the 29th Chinese Control and Decision Conference, Chongqing, China, 28-30 May 2017; pp. 5957-5960.

10. Liang, H.; Liu, Q.; Fang, Y. The measurement of thickness of cable aluminum protective jacket on line. J. Xi'an Univ. Technol. 1998, 14, 19-24.

11. Wen, J.; Zhao, H. On line measurement of cable insulation wall thickness and eccentricity using eddy current. In Proceedings of the 7th International Conference on Properties and Applications of Dielectric Materials, Nagoya, Japan, 1-5 June 2003; pp. 816-819.

12. Fan, C.; Zou, L.; Wang, Y. Digital image processing techniques applied in cable insulation parameters measurement. In Proceedings of the 2008 IEEE International Conference on Automation and Logistics, Qingdao, China, 1-3 September 2008; pp. 2315-2319.

13. Shan, B.; Wang, Y.; Fan, C. Research of measure system to cable insulation parameters based on computer vision. In Proceedings of the 2009 First International Workshop on Education Technology and Computer Science, Wuhan, China, 7-8 March 2009; pp. 776-779.

14. Bian, J.H.; Wang, J.L.; Xu, S.; Zhou, T. A cable sheath material thickness measurement method based on image measurement technology. In Applied Mechanics and Materials; SJR: Stafa-Zurich, Switzerland, 2012; Volume 182-183, pp. 477-481.

15. Xia, S. Sub-pixel measurement of the cable sheath materials thickness. In Applied Mechanics and Materials; SJR: Stafa-Zurich, Switzerland, 2013; Volume 333-335, pp. 1047-1050.

16. Cheng, H.; Zhai, Y.; Chen, R.; Wang, D.; Dong, Z.; Wang, Y. Self-shattering defect detection of glass insulators based on spatial features. Energies 2019, 12, 543. [CrossRef]

17. Siddiqui, Z.A.; Park, U.; Lee, S.; Jung, N.; Choi, M.; Lim, C.; Seo, J. Robust powerline equipment inspection system based on a convolutional neural network. Sensors 2018, 18, 3837. [CrossRef] [PubMed]

18. Hao, Y.; Wei, J.; Jiang, X.; Yang, L.; Li, L.; Wang, J.; Li, H.; Li, R. Icing condition assessment of in-service glass insulators based on graphical shed spacing and graphical shed overhang. Energies 2018, 11, 318. [CrossRef] 
19. Fard, M.; Farrag, M.; McMeekin, S.; Reid, A. Electrical treeing in cable insulation under different HVDC operational conditions. Energies 2018, 11, 2406. [CrossRef]

20. Kang, S.; Sim, B.; Kim, J. Volume and mass measurement of a burning wood pellet by image processing. Energies 2017, 10, 603. [CrossRef]

21. Lee, J.; Lee, K.; Cho, S.; Sim, S. Computer vision-based structural displacement measurement robust to light-induced image degradation for in-service bridges. Sensors 2017, 17, 2317. [CrossRef]

22. Zhang, X.; Zhang, J.; Ma, M.; Chen, Z.; Yue, S.; He, T.; Xu, X. A high precision quality inspection system for steel bars based on machine vision. Sensors 2018, 18, 2732. [CrossRef]

23. Yao, Z.; Yi, W. Curvature aided hough transform for circle detection. Expert Syst. Appl. 2016, 51, 26-33. [CrossRef]

24. Olijve, L.L.C.; Oude Vrielink, A.S.; Voets, I.K. A simple and quantitative method to evaluate ice recrystallization kinetics using the circle hough transform algorithm. Cryst. Growth Des. 2016, 16, 4190-4195. [CrossRef]

25. Djekoune, A.O.; Messaoudi, K.; Amara, K. Incremental circle hough transform: An improved method for circle detection. Optik 2017, 133, 17-31. [CrossRef]

26. Kim, H.; Kim, J. A two-step circle detection algorithm from the intersecting chords. Pattern Recognit. Lett. 2001, 22, 787-798. [CrossRef]

27. Andres, E.; Largeteau-Skapin, G.; Rodríguez, M. Generalized perpendicular bisector and exhaustive discrete circle recognition. Graph. Models 2011, 73, 354-364. [CrossRef]

28. Cai, B.; Wang, Y.; Wang, K.; Ma, M.; Chen, X. Camera calibration robust to defocus using phase-shifting patterns. Sensors 2017, 17, 2361. [CrossRef] [PubMed]

29. Achler, O.; Trivedi, M.M. Camera based vehicle detection, tracking, and wheel baseline estimation approach. In Proceedings of the 7th International IEEE Conference on Intelligent Transportation Systems, Washington, WA, USA, 3-6 October 2004; pp. 743-748.

30. Qian, R.; Zhang, B.; Yue, Y.; Coenen, F. Traffic sign detection by template matching based on multi-level chain code histogram. In Proceedings of the 12th International Conference on Fuzzy Systems and Knowledge Discovery (FSKD), Zhangjiajie, China, 15-17 August 2015; pp. 2400-2404.

31. Odagiri, M.; Onoguchi, K. Circle detection based on arc search using a table of virtual circle. In Proceedings of the 2016 International Conference on Digital Image Computing: Techniques and Applications (DICTA), Gold Coast, Australia, 30 November-2 December 2016; pp. 1-6.

32. Cuevas, E.; Oliva, D.; Zaldivar, D.; Pérez-Cisneros, M.; Sossa, H. Circle detection using electro-magnetism optimization. Inf. Sci. 2012, 182, 40-55. [CrossRef]

33. Cuevas, E.; Zaldivar, D.; Perez, M.; Ramirez, M. Circle detection using discrete differential evolution optimization. Pattern Anal. Appl. 2011, 14, 93-107. [CrossRef]

34. Brown, D.C. Close-range camera calibration. Photogramm. Eng. 1971, 37, 855-866.

35. Zhang, Z. A flexible new technique for camera calibration. IEEE Trans. Pattern Anal. Mach. Intell. 2000, 22, 1330-1334. [CrossRef]

36. Budil, D.E.; Lee, S.; Saxena, S.; Freed, J.H. Nonlinear-Least-Squares Analysis of Slow-Motion EPR Spectra in One and Two Dimensions Using a Modified Levenberg-Marquardt Algorithm. J. Magn. Reson. Ser. A 1996, 120, 155-189. [CrossRef]

37. Bradski, G.; Kaehler, A. Learning OpenCV: Computer Vision with the OpenCV Library, 1st ed.; O'Reilly Media: Sebastopol, CA, USA, 2009; pp. 370-397.

38. International Telecommunication Union. ITU-R Recommendation BT.601; Radiocommunication Sector of ITU: Zeneva, Switzerland, 2017.

39. Canny, J. A computational approach to edge detection. IEEE Trans. Pattern Anal. Mach. Intell. 1986, 8, 679-698. [CrossRef] [PubMed]

40. Douglas, D.H.; Peucker, T.K. Algorithms for the reduction of the number of points required to represent a digitized line or its caricature. Cartogr. Int. J. Geogr. Inf. Geov. 1973, 10, 112-122. [CrossRef]

41. Barber, C.; Dobkin, D.; Huhdanpaa, H. The quickhull algorithm for convex hulls. ACM Trans. Math. Softw. 1996, 22, 469-483. [CrossRef]

(C) 2019 by the authors. Licensee MDPI, Basel, Switzerland. This article is an open access article distributed under the terms and conditions of the Creative Commons Attribution (CC BY) license (http://creativecommons.org/licenses/by/4.0/). 